ARTICLE

\title{
PNPLA1 has a crucial role in skin barrier function by directing acylceramide biosynthesis
}

Tetsuya Hirabayashi ${ }^{1,2}{ }^{\star}$, Tatsuki Anjo ${ }^{1,3, \star}$, Arisa Kaneko ${ }^{1,4}$, Yuuya Senoo ${ }^{5}$, Akitaka Shibata ${ }^{6}$, Hiroyuki Takama ${ }^{6}$ Kohei Yokoyama7, Yasumasa Nishito7, Tomio Ono ${ }^{7}$, Choji Taya ${ }^{7}$, Kazuaki Muramatsu ${ }^{3}$, Kiyoko Fukami ${ }^{4}$, Agustí Muñoz-Garcia ${ }^{8}$, Alan R. Brash ${ }^{9}$, Kazutaka Ikeda ${ }^{5}$, Makoto Arita ${ }^{5}$, Masashi Akiyama ${ }^{6}$ \& Makoto Murakami ${ }^{1,2}$

Mutations in patatin-like phospholipase domain-containing 1 (PNPLA1) cause autosomal recessive congenital ichthyosis, but the mechanism involved remains unclear. Here we show that PNPLA1, an enzyme expressed in differentiated keratinocytes, plays a crucial role in the biosynthesis of $\omega$-O-acylceramide, a lipid component essential for skin barrier. Global or keratinocyte-specific Pnpla1-deficient neonates die due to epidermal permeability barrier defects with severe transepidermal water loss, decreased intercellular lipid lamellae in the stratum corneum, and aberrant keratinocyte differentiation. In Pnpla1-/- epidermis, unique linoleate-containing lipids including acylceramides, acylglucosylceramides and (O-acyl)- $\omega$-hydroxy fatty acids are almost absent with reciprocal increases in their putative precursors, indicating that PNPLA1 catalyses the $\omega$-O-esterification with linoleic acid to form acylceramides. Moreover, acylceramide supplementation partially rescues the altered differentiation of Pnpla1-/- keratinocytes. Our findings provide valuable insight into the skin barrier formation and ichthyosis development, and may contribute to novel therapeutic strategies for treatment of epidermal barrier defects.

\footnotetext{
${ }^{1}$ Lipid Metabolism Project, Tokyo Metropolitan Institute of Medical Science, Tokyo 156-8506, Japan. ${ }^{2}$ AMED-CREST, Japan Agency for Medical Research and Development, Tokyo 100-0004, Japan. ${ }^{3}$ Division of Life Science and Engineering, School of Science and Engineering, Tokyo Denki University, Saitama 3500394, Japan. ${ }^{4}$ Laboratory of Genome and Biosignals, Tokyo University of Pharmacy and Life Sciences, Tokyo 192-0392, Japan. ${ }^{5}$ Laboratory for Metabolomics, RIKEN Center for Integrative Medical Sciences, Kanagawa 230-0045, Japan. ${ }^{6}$ Department of Dermatology, Nagoya University Graduate School of Medicine, Aichi 466-8550, Japan. ${ }^{7}$ Center for Basic Technology Research, Tokyo Metropolitan Institute of Medical Science, Tokyo 156-8506, Japan. ${ }^{8}$ Department of Evolution, Ecology, and Organismal Biology, Ohio State University, Ohio 43210, USA. ${ }^{9}$ Vanderbilt Institute of Chemical Biology, Vanderbilt University,

Nashville, Tennessee 37232-6304, USA. * These authors contributed equally to this work. Correspondence and requests for materials should be addressed to M.M. (email: murakami-mk@igakuken.or.jp).
} 
T he skin barrier of terrestrial mammals is essential for prevention of water and electrolyte loss, as well as protection from the penetration of harmful substances and pathogenic microbes ${ }^{1,2}$. Impairment of skin barrier function can cause or aggravate skin disorders, including dry skin, ichthyosis, psoriasis, and atopic dermatitis ${ }^{3-7}$. Although the epidermis is a highly organized stratified epithelium consisting of four distinctive layers, the innermost stratum basale (SB), the stratum spinosum (SS), the stratum granulosum (SG) and the uppermost stratum corneum (SC), its barrier function is provided mainly by specialized structures in the SC and tight junctions in the $\mathrm{SG}^{8,9}$. The unique $\mathrm{SC}$ components include cross-linked, insoluble proteins of corneocytes forming the cornified envelope (CE) and its associated, external membrane monolayer, called the cornified lipid envelope (CLE), as well as the intercellular lipid lamellae, which are mainly composed of ceramides, cholesterol and free fatty acids (FFAs) and are secreted as lamellar body lipids by differentiated keratinocytes at the SG/SC interface ${ }^{10-12}$.

Among the epidermal ceramides with marked molecular heterogeneity (at least 12 classes in humans) ${ }^{13,14}$, acylceramide is essential for physical and functional organization of lipids in the SC interstices, and thereby the barrier function of the $\operatorname{skin}^{1,3,11,15}$. Impaired biosynthesis or processing of acylceramide causes ichthyosis, characterized by dry, scaly and thickened skin. Acylceramide is an unusual ceramide species whose $\mathrm{N}$-acyl chain is composed of $\omega$-hydroxylated ultra-long chain FAs (ULCFAs) esterified at the $\omega$-position with linoleic acid (LA; C18:2). It has been suggested that the ULCFA portion of acylceramide spans a bilayer while the LA tail inserts into a closely apposed section of bilayer, thus serving as a molecular rivet to link two membranes together in the lipid lamellae ${ }^{16}$. In addition, acylceramides containing $\omega$-O-esterified fatty acids other than LA cannot be converted to covalently protein-bound $\omega$-hydroxyceramide (Cer OS), which forms CLE and functions as a template on the surfaces of corneocytes for the organization of lipid layers in the SC interstices. Indeed, in essential FA deficiency, LA in acylceramide is replaced by oleic acid, which fails to support skin barrier function properly ${ }^{17}$. Until now, several important steps for acyceramide biosynthesis and processing in the epidermis have been identified from studies of autosomal recessive congenital ichthyosis (ARCI) in humans and corresponding mouse disease models with genetic knockouts: the synthesis of ULCFAs by the FA elongase ELOVL4, $\omega$-hydroxylation of ULCFAs by the FA $\omega$-hydroxylase CYP4F22 (or CYP4F39 in mice), and formation of ceramides with ULCFAs by the ceramide synthase CERS3 (refs 18-20). However, the mechanism underlying the formation of acylceramide with LA in the epidermis is still under debate.

The current model for $\omega$-O-esterification of ULCFA-ceramides with LA involves the hydrolysis of triacylglycerol (TG) in lipid droplets to provide LA via a yet unknown lipase, followed by its transfer (via acyl-CoA form) to the $\omega$-hydroxy group of ULCFA in ceramides or glucosylceramides (GlcCer) by a putative $\omega$-O-acyltransferase. Alternatively, LA can be directly transferred from TG to $\omega-\mathrm{OH}$ Cer and/or $\omega-\mathrm{OH}$ GlcCer by an LA-specific transacylase ${ }^{8,21}$. Recent studies of patients with neutral lipid storage disease with ichthyosis (NLSDI or Chanarin-Dorfman syndrome) and in mice with Abhd5 deletion suggest that TG accumulation due to loss-of-function of ABHD5 (also known as CGI-58) reduces the availability of LA for acylceramide production 22,23 . ABHD5 is an essential co-factor for stimulation of ATGL (adipose triglyceride lipase, also known as PNPLA2 or iPLA ${ }_{2} \zeta$ ), which plays a major role in TG hydrolysis in most tissues ${ }^{24,25}$. ATGL is a member of the patatin-like phospholipase domain-containing protein (PNPLA) or $\mathrm{Ca}^{2+}$. independent phospholipase $\mathrm{A}_{2}\left(\mathrm{iPLA}_{2}\right)$ family, which comprises 9 enzymes in humans acting as lipid hydrolases, acyltransferases or transacylases with diverse substrate specificities including phospholipids and neutral lipids ${ }^{26}$. Interestingly, ichthyosis features and decreased acylceramide levels in the skin have been observed in patients and mice with defective ABHD5 function, but not in those with ATGL mutations or deletion, leading to the proposal that ABHD5 could activate a different lipase that regulates epidermal TG hydrolysis ${ }^{21,25}$. Nonetheless, the molecular entity of $\omega$-O-acyltransferase or transacylase responsible for the linoleoyl $\omega$-O-esterification of ULCFAceramides has not yet been identified.

Loss-of-function mutations in PNPLA1, a paralog of ATGL/PNPLA2, have recently been identified in humans or dogs with ARCI (refs 27-29). PNPLA1 fails to hydrolyse TG, however, even in the presence of ABHD5 (ref. 29), raising the question of the role of this functionally orphan enzyme. How lipid metabolism regulated by PNPLA1 contributes to epidermal homoeostasis is a fundamental issue which remains to be addressed. To this end, we herein generated Pnpla1-deficient mice in our ongoing efforts to decipher the biological roles of $\mathrm{PLA}_{2}$-related enzymes by gene targeting ${ }^{30-33}$. We provide evidence that absence of PNPLA1 causes a severe skin permeability barrier defect by perturbing the linoleoyl $\omega$-O-esterification of ceramides to yield acylceramides, along with abnormal differentiation of keratinocytes, thus demonstrating that PNPLA1 is a long-sought enzyme that plays a critical role in acylceramide synthesis in the skin.

\section{Results}

Expression of PNPLA1 in highly differentiated keratinocytes. Among adult mouse tissues, Pnpla1 messenger RNA (mRNA) was expressed most abundantly in the skin, followed by the stomach (Supplementary Fig. 1a). Immunohistochemistry of newborn mouse skin revealed localization of PNPLA1 protein in the boundary area between the nucleated SG and the denucleated SC, just above the location of the granular layer marker loricrin, in the epidermis (Supplementary Fig. 1b). In agreement with a previous report ${ }^{29}$, PNPLA1 was partially colocalized with filaggrin (a SG marker), but not with keratin 1 and 5 (SS and SB markers, respectively) (Supplementary Fig. 1b). In adult mouse skin, the localization of PNPLA1 in the epidermis was essentially the same as that in newborn skin (Supplementary Fig. 1c). In a monolayer culture of mouse keratinocytes, $\mathrm{Ca}^{2+}$ treatment resulted in marked induction of keratinocyte differentiation markers (Krt10 and Lor), as well as Pnpla1 (Supplementary Fig. 1d). Likewise, a marked increase of PNPLA1 expression was observed in human keratinocytes after $\mathrm{Ca}^{2+}$-induced differentiation, an event that occurred in parallel with induced expression of the keratinocyte differentiation markers $K R T 1$ and $F L G$, but not with constitutive expression of the SB marker KRT5 (Supplementary Fig. 1e). These results suggest that PNPLA1 has a specific role in highly differentiated keratinocytes in the uppermost layer of the SG, where lipids required for epidermal barrier function are processed and secreted into the intercellular space to form lipid lamellae and CLE.

Impaired epidermal permeability barrier in Pnpla1 ${ }^{-1-}$ mice. To gain insight into the function of PNPLA1 in vivo, we generated mice with targeted disruption of the Pnplal gene on a C57BL/6 background (Supplementary Fig. 2a,b). The absence of mRNA and protein for PNPLA1 in the skin of Pnpla1 ${ }^{-1-}$ mice was confirmed by quantitative PCR (qPCR) (Supplementary Fig. 2c) and immunohistochemistry (Fig. 1a), respectively. Offspring from heterozygote intercrosses were born at the 
a

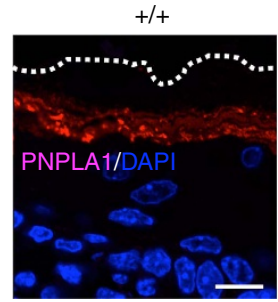

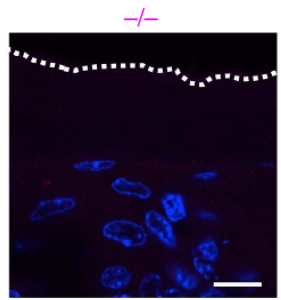

b

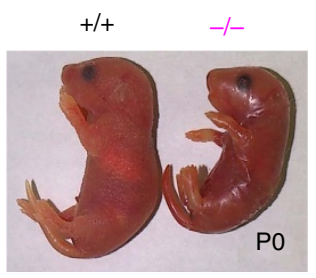

C

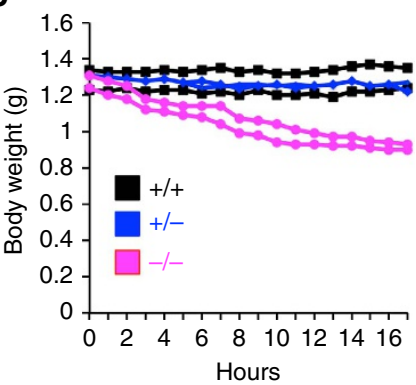

d

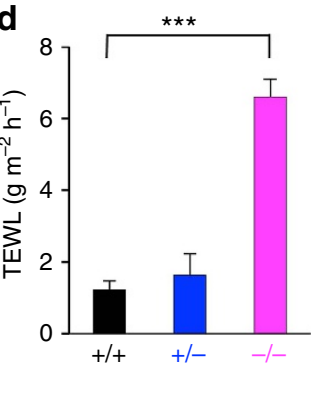

e

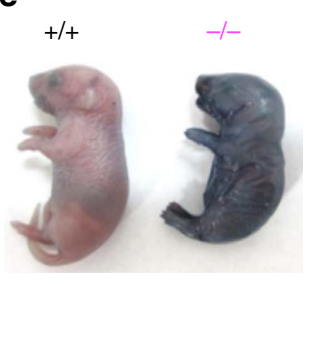

g
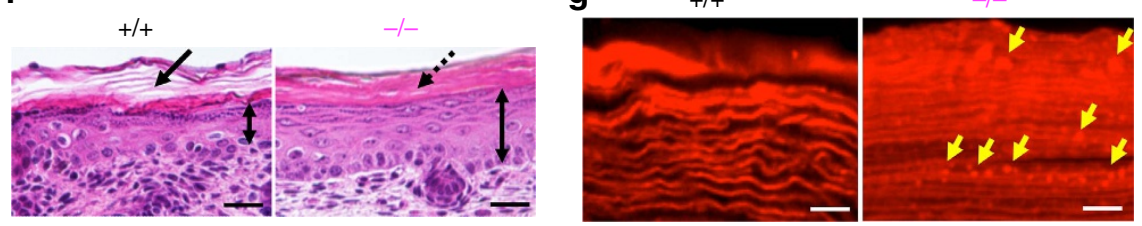

$\mathbf{h}$

$+/+$


i
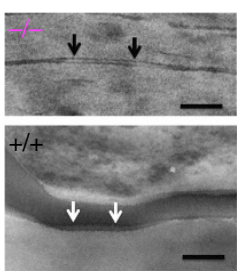

Figure 1 | Impaired skin barrier function in Pnpla1 ${ }^{-1-}$ mice. (a) Immunohistochemical staining for PNPLA1 (red), followed by counterstaining with DAPI (blue), in skin sections from Pnpla1 ${ }^{+/+}$and Pnpla1 ${ }^{-/-}$newborns. Dashed lines indicate the upper border of the epidermis. (b) Gross appearance of Pnpla1 ${ }^{+/+}$and Pnpla1 ${ }^{-/-}$newborns at PO. (c) Monitoring of body weights of Pnpla1 ${ }^{+/+}$, Pnpla1 ${ }^{+} /-$and Pnpla1 ${ }^{-/-}$mice (two pups for each) after Caesarean section at E18.5. (d) Skin permeability as assessed by TEWL on the dorsal skin surface of Pnpla1 ${ }^{+/+}(n=19), P_{n p l a 1^{+/}-(n=21)}$ and Pnpla1 ${ }^{-1-}$ mice $(n=21)$ (mean \pm s.e.m., ${ }^{\star \star \star} P<0.001$ in an unpaired, two-tailed Student's $t$-test). (e) Toluidine blue exclusion assay using neonatal Pnpla1 ${ }^{+/+}$and Pnpla1 ${ }^{-/-}$mice. (f) Histology of dorsal skin sections from newborn Pnpla $1^{+/+}$and Pnpla1 ${ }^{-/-}$mice stained with hematoxylin and eosin. Arrow indicates a basket weave structure in the SC of WT mice. Mutant epidermis was notably thicker (double-headed arrows) and had a more tightly packed SC (dashed arrow) than WT epidermis. (g) Staining of SC lipids with Nile red. Continuous linear lipid structures in the SC of Pnpla1 $+/+$ mice were replaced by a dot-like pattern (yellow arrows) in the SC of Pnpla $1^{-/-}$mice. (h,i) Transmission electron microscopy of skins of Pnpla ${ }^{+/}+$and Pnpla ${ }^{-/}-$ newborn mice. Compared with Pnpla1 ${ }^{+/+}$skin, Pnpla1 ${ }^{-/-}$skin displayed numerous lipid aggregates in corneocytes (blue boxes), abnormalites in the secreted contents at the SG-SC interface (red circles) (h), and impaired formation of the CLE (arrows) (i). Scale bars; $20 \mu \mathrm{m}(\mathbf{a}, \mathbf{f}), 5 \mu \mathrm{m}(\mathbf{g}), 0.4 \mu \mathrm{m}$ (h) and $0.2 \mu \mathrm{m}$ (i). Representative $(\mathbf{a}-\mathbf{c}, \mathbf{e}-\mathbf{i})$ or complied (d) results from at least three experiments are shown.

expected Mendelian ratio (Supplementary Fig. 2d). Although Pnpla $1^{+1-}$ mice were healthy and indistinguishable from Pnpla1 $1^{+1+}$ mice, newborn Pnpla1 ${ }^{-1-}$ pups had shiny and taut skin, often with a necrotic tail tip (Fig. 1b; Supplementary Fig. 2e), and died within $24 \mathrm{~h}$ after birth. We hypothesized that the cause of death in these Pnpla1-deficient mice might be dehydration, and therefore we assessed their skin permeability barrier function. Pnpla $1^{-1}$ pups delivered by Caesarean section at E18.5 had normal body weight as compared with littermate wild-type (WT) and heterozygous mice at birth, but rapidly lost as much as $20 \%$ of their weight within $16 \mathrm{~h}$ (Fig. 1c). In accordance with this steep weight loss, trans-epidermal water loss (TEWL) was markedly higher in Pnpla1 $1^{-/-}$newborns than in Pnpla1 ${ }^{+/+}$and
Pnpla1 ${ }^{+/-}$newborns (Fig. 1d), indicating a severe defect of the inside-out barrier in the null mice. In the toluidine blue exclusion assay to assess the outside-in permeability barrier, WT littermates excluded dye, whereas Pnpla1 ${ }^{-1-}$ pups showed robust dye penetration into the skin (Fig. 1e). These phenotypes, which have been commonly observed in mutant mice with disruption of genes associated with ARCI (ref. 1), suggest that PNPLA1 is required for epidermal permeability barrier function.

Histological analysis revealed that control mice had a clear basket weave-like structure segregated by interspaces, indicative of the presence of lipid lamellae (Fig. 1f, left). In contrast, Pnpla1 ${ }^{-/-}$mice exhibited a tightly packed structure in the SC, a reduced number of keratohyaline granules in the uppermost SG, 
and epidermal hyperplasia (Fig. 1f, right), which is considered to be an adaptive response to barrier disruption. Nile red staining of the Pnpla $1^{+/+}$epidermis showed wavy lipid multilayers characteristic of SC intercellular lipid lamellae, whereas granular-like lipid aggregates were present within increased number of densely packed lamellar sheets in the Pnpla $1^{-1-}$ epidermis (Fig. 1g), suggesting that keratinocytes are hyperproliferative and defective in the secretion and/or composition of SC lipids in mutant mice. Ultrastructural examination of Pnpla $1^{-1-}$ mice by transmission electron microscopy confirmed the tightly stacked layers of corneocytes with a substantially decreased amount of intercellular lipid lamellae, as evidenced by narrowed SC interstices, in comparison with control mice (Fig. 1h). At the SG-SC interface of Pnpla1 ${ }^{+1+}$ epidermis, lipid lamellae were released into the intercellular spaces from lamellar bodies (Fig. 1h). In contrast, the secretion of lipid granule contents was hampered and abnormal vesicular structures, which were thought to represent defective lamellar bodies, were retained within corneocytes in Pnpla1 ${ }^{-1}$ - epidermis (Fig. 1h). Moreover, Pnpla $1^{-/-}$mice exhibited either loss or abnormalities of the CLE (Fig. 1i). These results indicate that PNPLA1 plays a critical role in the proper formation of intercellular lipid lamellae and CLE in the SC, which are important for the permeability barrier function of the skin.

Aberrant keratinocyte differentiation in Pnpla1 ${ }^{-1-}$ mice. To further address the skin abnormalities in Pnpla1 ${ }^{-1-}$ mice, we performed microarray gene profiling using skins of newborn Pnpla1 ${ }^{+/+}$and Pnpla1 ${ }^{-/-}$mice. Heat map visualization of selected genes indicated down-regulation of genes for late keratinocyte differentiation and CE constituents (for example, Lor, Flg, Flg2, and the late cornified envelope genes Lce1a, b) in Pnpla1 ${ }^{-1}-$ epidermis (Supplementary Fig. 3a). Most of these are located within the epidermal differentiation complex, a keratinocyte lineage-specific gene locus on mouse chromosome 3. Several up-regulated gene clusters within the epidermal differentiation complex, such as small proline-rich proteins (Sprr1a, Sprr1b and Sprr2b), late cornified envelope proteins (Lce3b and Lce3c), and S100 proteins (S100a8 and S100a9), have been associated with psoriasis ${ }^{34}$. Other up-regulated genes included those involved in keratinocyte proliferation linked to epidermal growth factor (EGF) signalling (for example, Areg, Epgn, Tgfa, Hbegf and Ereg), adhesive structures (for example, Cldn4, Cldn7, Dsc1, Dsc2, Ocln, Tjp1 and Tjp2), lipid metabolism (for example, Fasn, Scd1, Pla2g4d and Pla2g4e) and skinassociated immune responses (for example, Il1b, Il12a, Il13, Il20, Il22, Il23a, Tnf, Ifng and Cxcl1) (Supplementary Fig. 3a). It is likely that the enhanced expression of inflammatory cytokines and chemokines is a secondary effect resulting from impaired barrier function, since similar changes have also been observed in several genetically distinct mouse models with barrier defects ${ }^{33-38}$ and patients with skin diseases such as ichthyosis, atopic dermatitis and psoriasis ${ }^{39,40}$. Interestingly, expression levels of genes associated with synthesis and processing of epidermal acylceramide were consistently elevated in Pnpla1 ${ }^{-}-$mice relative to Pnpla1 ${ }^{+1+}$ and Pnpla1 ${ }^{+/-}$mice (Supplementary Fig. 3a,b). These genes included Elovl4, Abhd5, Cers3, Cyp4f39 (a mouse ortholog of human CYP4F22), Ugcg, Abca12 and Gba, mutation or deletion of which has been shown to cause ARCI in humans and neonatal death in mice due to severe skin barrier defects $^{1,41}$.

Immunofluorescence staining and qPCR confirmed the diminished expression of terminal differentiation markers, such as filaggrin (Flg) and loricrin (Lor), in Pnpla1 ${ }^{-1-}$ skin relative to Pnpla1 $+1+$ skin, whereas mRNA and protein expression levels of the basal and early suprabasal keratinocyte differentiation markers, keratin $5(K r t 5)$ and $1(K r t 1)$, were similar between the two genotypes (Fig. 2a,b). In contrast, keratin 6 (Krt6a and $K r t 6 b)$ was expressed in the lower suprabasal layer in Pnpla1deficient but not in control skin, reflecting the hyperproliferative state of the mutant epidermis. Abnormal differentiation of keratinocytes has also been observed in several mouse lines with targeted disruption of genes implicated in epidermal ceramide metabolism ${ }^{22,35}$. Therefore, the neonatal lethality of Pnpla1 ${ }^{-1-}$ mice due to skin barrier defect is likely dependent upon both altered lipid composition and impaired differentiation of keratinocytes.

Moreover, expression of PPAR $\delta$ (Ppard) and its potential target genes such as Fabp5 and Sprr1b (ref. 35) was markedly increased in Pnpla1 ${ }^{-1-}$ skin relative to WT skin (Fig. 2c; Supplementary Fig. 3a), indicating that PNPLA1 deficiency leads to hyperactivation of $\mathrm{PPAR} \delta$. Activation of EGF receptors has been shown to control keratinocyte proliferation and differentiation with decreased expression of differentiation-related genes including filaggrin and loricrin ${ }^{42}$. Indeed, heparin-binding EGF-like growth factor (HB-EGF), a potent autocrine growth factor for keratinocytes and putative target gene of PPAR $\delta$ (ref. 43), was robustly upregulated in Pnpla1 ${ }^{-/-}$epidermis (Fig. 2c; Supplementary Fig. 3a), suggesting that EGF receptor signalling contributes, at least in part, to epidermal hyperplasia and altered keratinocyte differentiation in the mutant mice.

Defective acylceramide biosynthesis in Pnpla1 ${ }^{-/-}$skin. To identify the endogenous lipid metabolism regulated by PNPLA1, we performed thin-layer chromatography (TLC) and quantitative liquid chromatography mass spectrometry (LC-MS/MS) using epidermal lipids extracted from neonatal WT and mutant mice. TLC analysis revealed that the bands for acylceramide (esterified omega-hydroxyacyl-sphingosine; EOS), which is a key determinant of skin permeability barrier function ${ }^{15}$, and its derivative acylglucosylceramide (GlcEOS) were markedly reduced or almost undetectable in Pnpla1 ${ }^{-1-}$ mice relative to WT and heterozygous mice (Fig. 3a). We also noticed that another lipid species, with a TLC motility slightly faster than that of FA, was nearly absent in mutant mice, and LC-MS/MS analysis with collision-induced fragmentation of this lipid extracted from the TLC plate identified it as (O-acyl)- $\omega$ hydroxy FA (OAHFA), particularly (O-linoleoyl)- $\omega$-hydroxy FA (OLHFA) (see below). In contrast, $\omega$-hydroxy FA ( $\omega$-OH FA), $\omega-\mathrm{OH} \mathrm{Cer}$ and GlcCer were present in substantially greater amounts in Pnpla1-I- mice than in control Pnpla1 $1^{+/+}$and Pnpla1 ${ }^{+/-}$mice (Fig. 3a).

To determine the changes in ceramide molecular species in terms of the length and saturation of their $N$-acyl chains, lipids extracted from Pnpla1 ${ }^{+/+}$and Pnpla1 ${ }^{-/-}$epidermis were analysed quantitatively by LC-MS and LC-MS/MS. Epidermal ceramide species are grouped into non-hydroxylated ceramides (NS, NDS, NH and NP), $\alpha$-hydroxylated ceramides (for example, AS, ADS, AH and AP) and acylceramides (for example, EOS, $\mathrm{EOH}$ and $\mathrm{EOP}$ ), where $\mathrm{S}, \mathrm{P}, \mathrm{DS}$ and $\mathrm{H}$ stand for sphingosine, phytosphingosine, dihydrosphingosine and 6-hydroxysphingosine, respectively ${ }^{44,45}$. EOS and EOP species with residues of (O-linoleoyl)- $\omega$-hydroxy ULCFAs (C28-C38) were almost entirely lost in the epidermis of Pnpla $1^{-1-}$ mice (Fig. 3b; quantitative data for representative molecular species are depicted in Supplementary Fig. 4a,b). Correspondingly, there was marked accumulation of various molecular species of $\omega-\mathrm{OH}$ Cer, a putative precursor of EOS, in mutant mice relative to WT mice (Fig. 3c). In addition, in mutant mice, the amounts of OLHFA species with C28-C36 ULCFAs were markedly decreased, with reciprocal increases in corresponding $\omega-\mathrm{OH}$ ULCFA species (Fig. 3d,e), confirming the results of TLC analysis (Fig. 3a). 
a
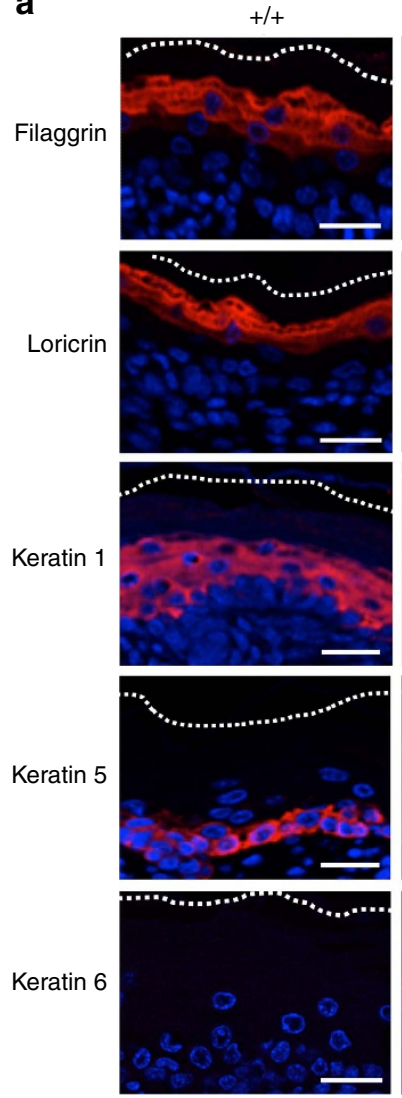

C

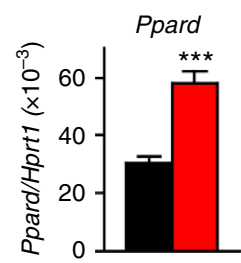

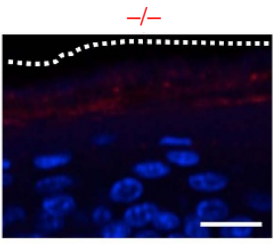
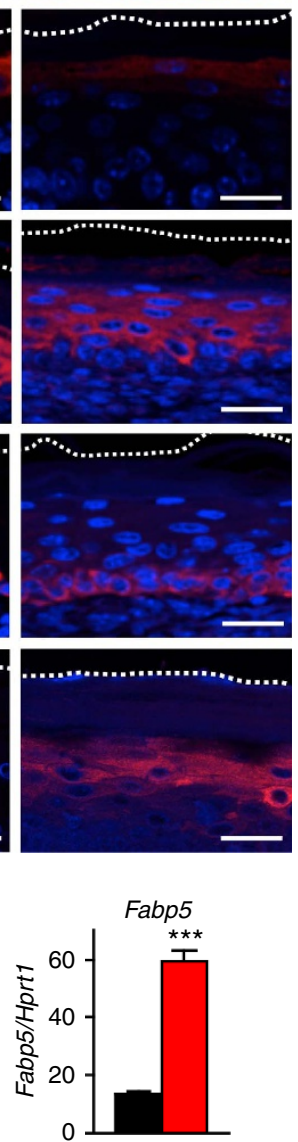

b
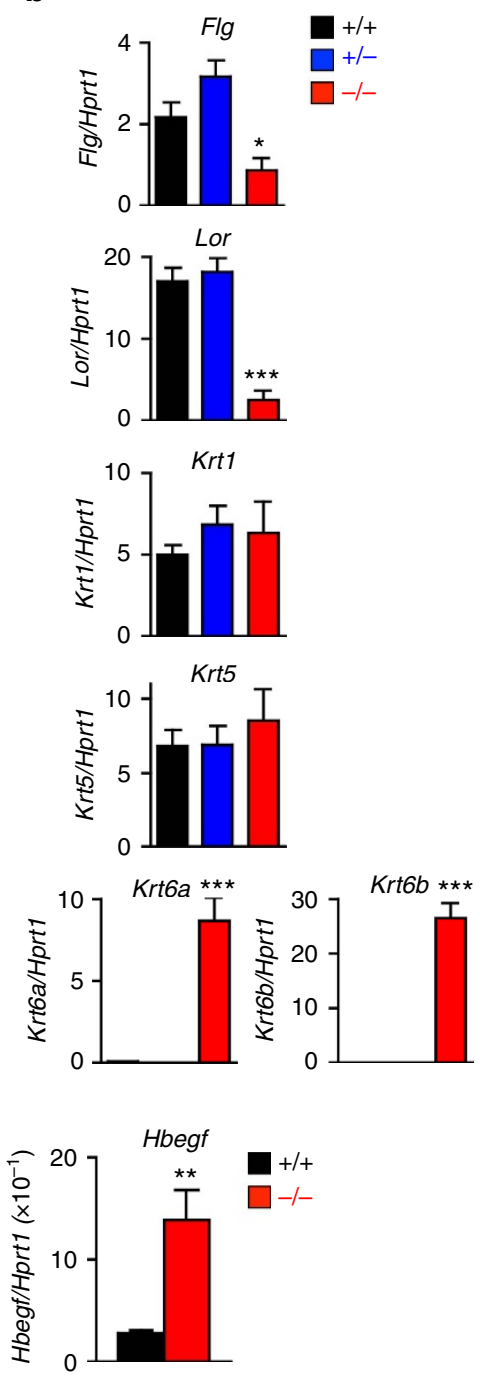

Figure 2 | Aberrant terminal differentiation of Pnpla1 ${ }^{-/-}$epidermis. (a) Immunohistochemical staining of keratinocyte differentiation markers (red), followed by conterstaining with DAPI (blue), in skin sections from Pnpla1 ${ }^{+/+}$and Pnpla1 ${ }^{-/-}$newborn mice. Scale bars, $20 \mu \mathrm{m}$. (b) qPCR analysis of keratinocyte differentiation markers in newborn Pnpla ${ }^{+/+}$, Pnpla1 ${ }^{+/-}$and Pnpla ${ }^{-/-}$epidermis ( $n=5$ animals per group). (c) qPCR analysis of PPAR $\delta$ (Ppard) and its potential target genes in newborn Pnpla ${ }^{+} /+$and Pnpla $1^{-/-}$epidermis $\left(n=7\right.$ animals). In b,c, values are mean \pm s.e.m.; ${ }^{\star} P<0.05$, ${ }^{\star \star} P<0.01$, and ${ }^{\star \star \star} P<0.001$ versus $P$ npla ${ }^{+/+}$mice. Representative results from two or three independent experiments are shown.

Moreover, the amount of Cer OS covalently bound to the CE was robustly reduced in mutant mice relative to WT mice (Supplementary Fig. 4c). In contrast to the dramatic reductions of acylceramides and their downstream products, various ceramide molecular species (AS, AP, NS, NH and NP) were modestly increased in Pnpla1 ${ }^{-1}$ mice (Supplementary Fig. $4 \mathrm{~d}-\mathrm{h})$. Collectively, these data suggest that PNPLA1 is required for linoleoyl $\omega$-O-esterification of the free and/or ceramide-bound forms of $\omega$-OH ULCFA residues. Interestingly, the linoleate residue of several, if not all, EOS and OAHFA species was partially replaced by the palmitate or oleate residue in Pnpla $1^{-1-}$ mice (Fig. 3b,d), indicating that, in the absence of PNPLA1, another putative acyltransferase or transacylase with weak activity and broad substrate specificity may contribute to the synthesis of EOS and OAHFA with non-linoleate fatty acid (that is, palmitate or oleate).

Although the epidermal levels of total ceramides were similar in both genotypes, those of FFAs, cholesterol and TG were substantially higher in Pnpla1 ${ }^{-/-}$mice than in Pnpla1 ${ }^{+/+}$ mice (Fig. 3a; Supplementary Fig. 5a). Since a proper ratio of ceramides, FFAs, and cholesterol is crucial for formation of the SC lipid lamellae, altered proportion of these lipids may lead to their unusual aggregation, as seen in the Pnpla1 - / - SC (Fig. 1g). Among the FFAs, the levels of very long chain FAs (VLCFAs; $\geq \mathrm{C} 22: 0$ ), but not those of long chain FAs (LCFAs), were increased in Pnpla1 ${ }^{-1-}$ mice (Supplementary Fig. 5b-d). These increases in cholesterol, VLCFAs and several ceramide species resulting from Pnpla1 deficiency accorded with the elevated expression levels of genes related to lipid metabolism such as Himgcr, Elovl4 and Degs2 (Supplementary Fig. 3a,b), suggesting compensatory adaptation of the Pnpla $1^{-1}$ epidermis to the impaired acylceramide synthesis and barrier formation. Moreover, our observation that the free LA level was unchanged in Pnpla1 ${ }^{-1-}$ mice (Supplementary Fig. 5d) argues against the alternative idea that PLPLA1 acts as a TG lipase that supplies LA for $\omega$-O-esterification of ULCFA. Although the composition of phospholipids was not profoundly affected by Pnpla1 deficiency, some phosphatidylethanolamine (PE) species with polyunsaturated fatty acids, including LA, were present in slightly greater amounts in Pnpla1 ${ }^{-P_{-}}$than in Pnpla1 ${ }^{+/+}$mice 
a

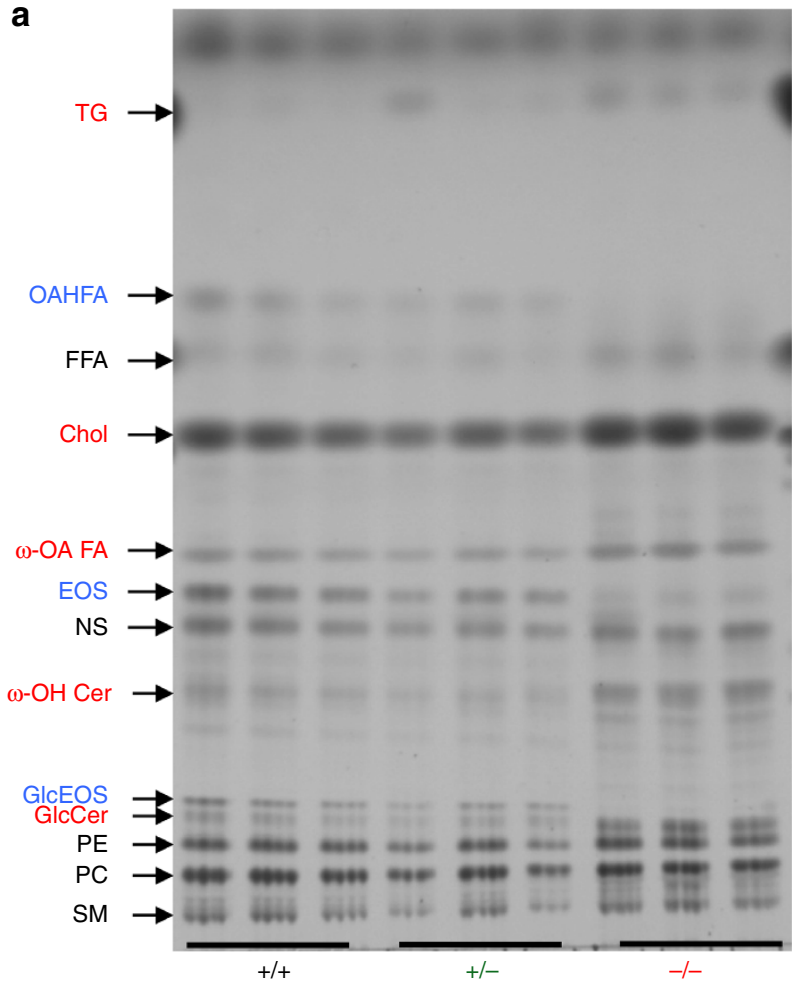

b

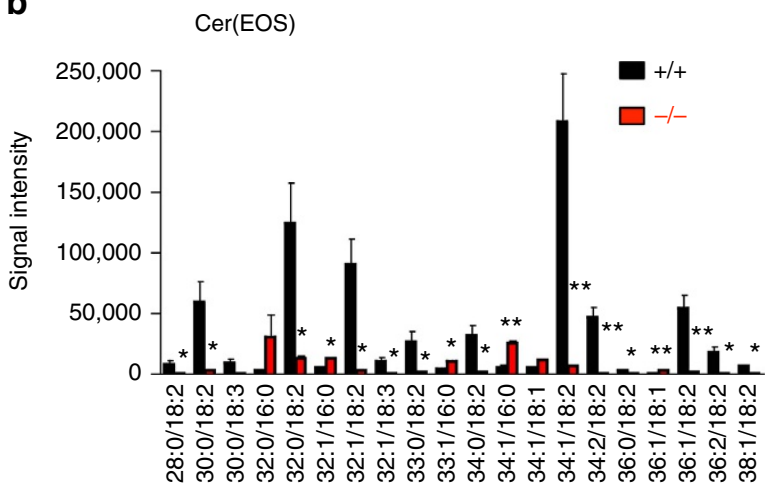

C

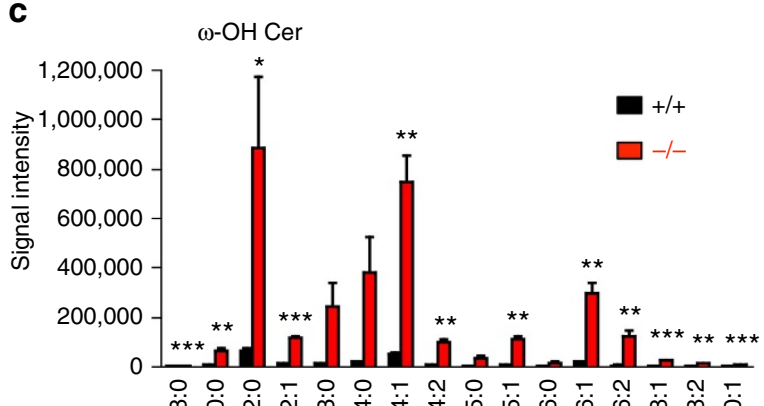

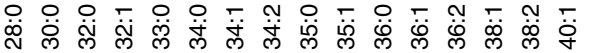

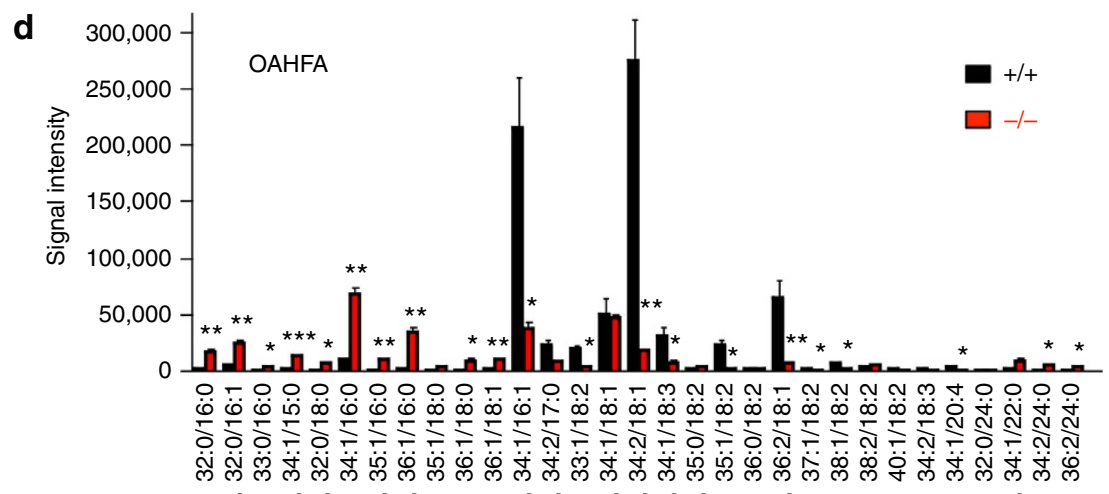

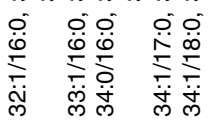

宓

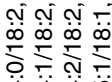

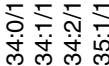

$\stackrel{\substack{\infty\\}}{\underset{0}{\infty}}$

守

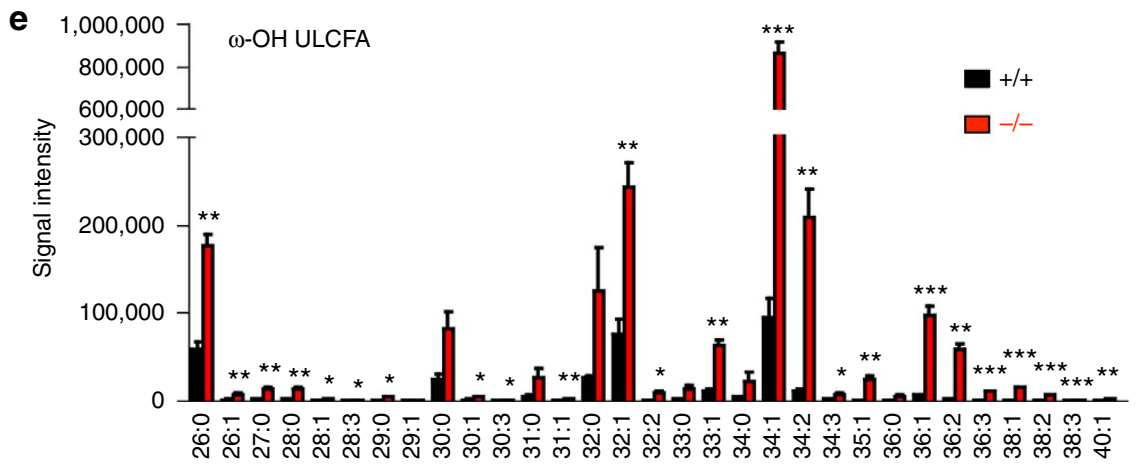

Figure 3 | Impaired acylceramide formation in Pnpla1 ${ }^{-/-}$epidermis. (a) Representative TLC analysis of lipids extracted from Pnpla1 ${ }^{+/+}$, Pnpla $^{+} /{ }^{-}$ and Pnpla1 ${ }^{-/-}$epidermis. In Pnpla1 -/ - mice, EOS, GlcEOS and OAFHA were almost completely depleted (blue), with reciprocal increases in $\omega$-OH FA, $\omega$ $\mathrm{OH}$ Cer and GlcCer (red), relative to Pnpla1 ${ }^{+/+}$and Pnpla1 ${ }^{+/-}$mice. TG, triglyceride; FFA, free fatty acid; Chol, cholesterol; PE,

phosphatidylethanolamine; PC, phosphatidylcholine: SM, sphingomyelin. (b-e) LC-MS/MS analysis of epidermal ceramide and related lipid species showing marked reductions in EOS (b) and OAHFA (d) species with linoleic acid (18:2) and increases in corresponding $\omega$-OH Cer (c) and $\omega$-OH ULCFA (e) species in Pnpla1 ${ }^{-/-}$mice in comparison with Pnpla1 ${ }^{+/+}$mice (mean \pm s.e.m., $n=3$ animals; ${ }^{\star} P<0.05,{ }^{\star \star} P<0.01$ and ${ }^{\star \star \star} P<0.001$ versus $P$ npla ${ }^{+/}++$ mice). In b,c, C18-sphingosine-based ceramide (d18:1) species are selected and shown. Results from one or two independent experiments are shown. 
(Supplementary Fig. 5e), probably because of the perturbed LA metabolism resulting from impaired formation of OLHFA and acylceramide.

Keratinocyte-specific Pnpla1 ablation impairs skin barrier. To ascertain whether the skin barrier defects observed in global Pnpla $1^{-1-}$ mice were indeed intrinsic to skin, mice carrying the loxP-flanked Pnpla1 allele (Pnpla1 f/f) were crossed with mice transgenic for Krt14 promoter-driven Cre recombinase to obtain mice lacking PNPLA1 selectively in epidermal keratinocytes (Pnpla1 ${ }^{f / f_{K}}$ 14-Cre). Expression of Pnpla1 in the skin was reduced by $\sim 80 \%$ in Pnpla1 ${ }^{f / f}$ K14-Cre mice in comparison with control Pnpla1 $^{f / f}$ mice (Fig. 4a), confirming that Cre-mediated recombination efficiently ablated Pnpla1 in epidermal keratinocytes.
About half reduction of Pnpla1 expression was also evident in the stomach, in which the $K 14$ promoter is active ${ }^{46}$, yet it is unlikely that this reduction could influence the skin phenotype since global heterozygous Pnpla1 ${ }^{+1-}$ mice showed no abnormality. Although Pnpla1 f/f K14-Cre animals were indistinguishable from control littermates shortly after birth, the mutant mice died within 6 days (Fig. 4b). The death was accompanied by focal desquamation with a markedly elevated TEWL value, whereas the value in other unaffected skin region remained unchanged (Fig. 4c,d). Histologically, a lower zone of the SC layers became densely packed with lipid-poor interspaces in Pnpla1 ${ }^{f / f_{K}} \mathrm{~K} 14-\mathrm{Cre}$ mice at P5 (Fig. 4e), as was seen in global Pnpla1-1- mice (Fig. 1f). Immunostaining of Pnpla1 f/f K14-Cre mice skin demonstrated diminished expression of filaggrin and loricrin in comparison to Pnpla1 ${ }^{f / f}$ mice (Fig. 4f). Furthermore, epidermal

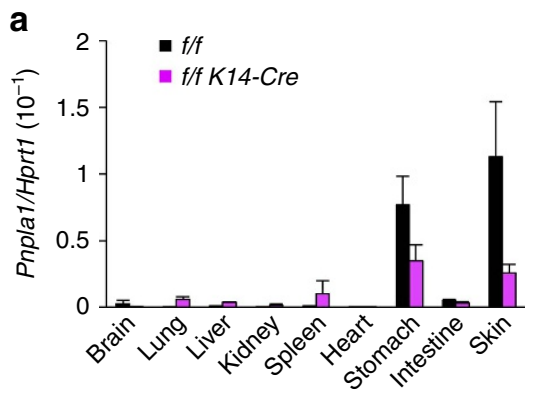

C

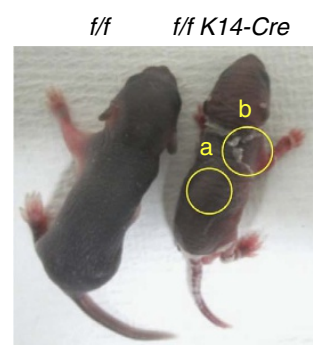

d

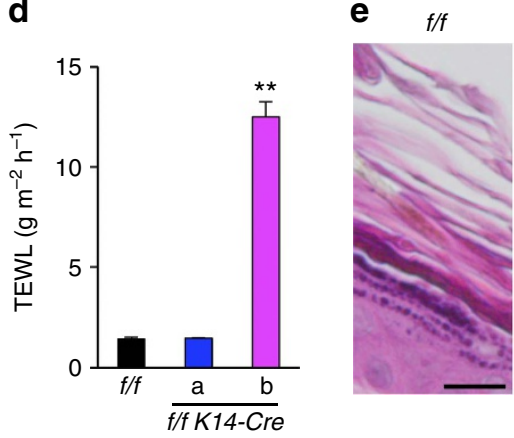

$\mathbf{f}$
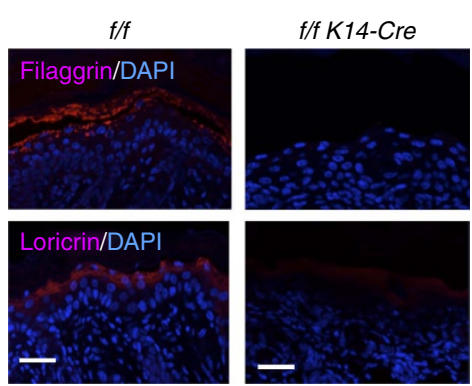

f/f K14-Cre

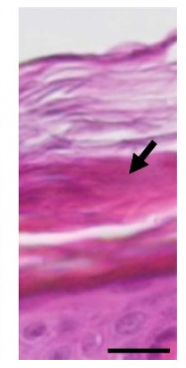

b

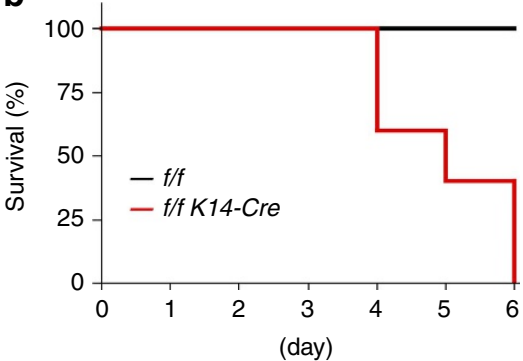

g

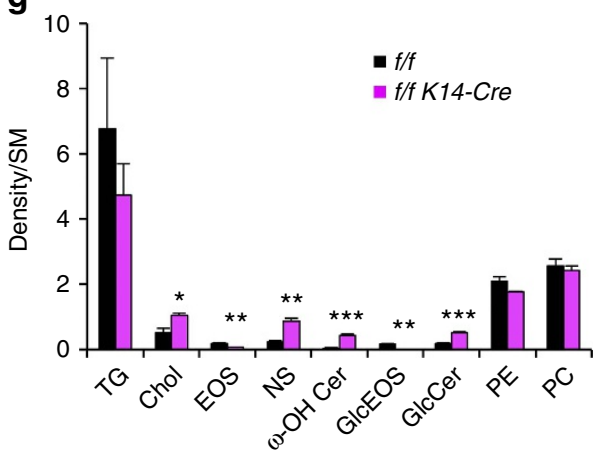

Figure 4 | Phenotypes of keratinocyte-specific Pnpla1-deficient mice. (a) qPCR analysis of Pnpla1 expression in various tissues of control $(f / f)(n=2)$ and Pnpla1 f/f K14-Cre ( $f / f$ K14-Cre) $(n=4)$ mice at P5. (b) Postnatal death within 6 days due to epidermal-specific disruption of Pnpla1 ( $n=5$ per genotype). (c) Gross appearance of control $(f / f)$ and mutant ( $f / f$ K14-Cre) mice at P5. Mutant animals showed smaller body size. Yellow circles labelled with a,b indicate regions without and with severe desquamation, respectively. (d) TEWL of control $(n=13)$ and mutant $(n=18)$ mice at P5. Labels a,b are as indicated in c. (e) Representative images of hematoxylin-eosin staining of skin sections from control and mutant mice at P5. In mutant mice, the lower part of the SC layers became densely packed with poor lipid interspaces (arrow). (f) Impaired terminal differentiation of epidermal keratinocytes at P5 in Pnpla $7^{f / f}$ K14-Cre mice. Sections were stained with anti-filaggrin and anti-loricrin antibodies (red) and DAPI (blue). (g) Densitometric analysis of TLC separation of epidermal lipids extracted from control and mutant mice at P5. Individual lipid levels were normalized with SM content ( $n=6$ animals). In $\mathbf{a}, \mathbf{d}, \mathbf{g}$, values are mean \pm s.e.m.; ${ }^{\star} P<0.05$, ${ }^{\star \star} P<0.01$ and ${ }^{\star \star \star} P P<0.001$ versus control mice. Scale bars in $\mathbf{e}, \mathbf{f}, 20 \mu \mathrm{m}$. Data are from at least two independent experiments. 
levels of EOS and GlcEOS were markedly lower, while those of $\omega$ $\mathrm{OH}$ Cer and GlcCer were conversely higher, in Pnpla ${ }^{f / f} \mathrm{~K} 14$-Cre mice (Fig. 4g). Taken together, these results suggest that PNPLA1 is required in a cell-autonomous manner for acylceramide formation and keratinocyte differentiation.

EOS rescues aberrant differentiation of mutant keratinocytes. To further investigate the function of PNPLA1 in keratinocyte differentiation, gene expression in primary keratinocytes prepared from Pnpla1 ${ }^{-1-}$ and control mice was analysed in culture. Consistent with the in vivo data (Fig. 2a-c), expression of the terminal differentiation marker Flg was lower in differentiated Pnpla $^{-1-}$ keratinocytes than in replicate control cells, while that of Ppard or Hbegf was significantly elevated in differentiated Pnpla1 $^{-1-}$ keratinocytes (Fig. 5). Supplementation of the differentiation medium with $\mathrm{EOS}(\mathrm{C} 30: 0)$ partially reversed the altered expression of Flg, Ppard and Hbegf in Pnpla1 ${ }^{-1-}$ keratinocytes (Fig. 5). These results suggest that the PNPLA1 product EOS or its derivative(s) regulates terminal keratinocyte differentiation partly through modulating PPAR $\delta$ expression.

Epidermal lipid composition in Pnpla1 ${ }^{-1-}$ and $A b h d 5^{-1-}$ mice. Last, we compared epidermal lipid composition between mouse lines deficient in Pnpla1 and Abhd5 (Cgi58, a co-factor for a putative TG lipase), both of which appear to converge on the processing of acylceramides 22 . Abhd $5^{-1-}$ epidermis at E18.5 showed partial reductions of OAHFA and EOS, an almost total depletion of GlcEOS, substantial increases of $\omega-\mathrm{OH}$ Cer and GlcCer, and marked accumulation of TG (Fig. 6a-c). These lipid profiles in $A b h d 5^{-1-}$ mice were similar to those in Pnpla1 ${ }^{-1-}$ mice, except that TG accumulation was not evident in the latter. Although the increase in TG content in Pnpla1 ${ }^{-1-}$ epidermis at P0 may be explained by the induction of lipogenic enzymes (Fig. 3a; Supplementary Fig. 3a), the distinct impact of $A b h d 5$ and Pnpla1 ablations on TG levels at E18.5 lends further support to segregation of PNPLA1 from bulk TG hydrolysis in which ABHD5 participates. Nonetheless, the similar reductions of OAHFA, EOS and GlcEOS in both Abhd5 ${ }^{-1-}$ and Pnpla1 ${ }^{-1-}$ mice support the cooperative roles of ABHD5 and PNPLA1 in the process of $\omega$-O-esterification; ABHD5 in assisting the bulk release of FFAs including LA from TG in lipid droplets and PNPLA1 in esterifying part of this LA pool into the free and/or ceramide-bound forms of $\omega-\mathrm{OH}$ FAs as an acyltransferase or transacylase. Overall, our results provide unequivocal evidence that PNPLA1 is a longsought enzyme responsible for $\omega$-O-esterification in acylceramide biosynthesis leading to proper formation of SC lamellae, keratinocyte differentiation, and thereby skin barrier function (Fig. 6d).

\section{Discussion}

It is generally known that $\mathrm{PLA}_{2}$ is a group of enzymes that hydrolyse the $s n-2$ position of glycerophospholipids to give rise to fatty acids and lysophospholipids. In fact, by hydrolyzing glycerophospholipids, cytosolic $\mathrm{PLA}_{2} \alpha$ plays a central role in arachidonic acid metabolism in a wide variety of cells, secreted $\mathrm{PLA}_{2} \mathrm{~s}$ modulate tissue-specific homoeostasis or diseases in given extracellular microenvironments, and PNPLA9 $\left(\mathrm{iPLA}_{2} \beta\right)$ and PNPLA8 ( $\mathrm{iPLA}_{2} \gamma$ ) participate in energy metabolism and neurodegeneration ${ }^{26,47}$. However, it has recently become obvious that several members of the PNPLA/iPLA 2 family catalyse forms of lipid metabolism other than the typical $\mathrm{PLA}_{2}$ reaction, as exemplified by PNPLA2/ATGL $\left(\mathrm{iPLA}_{2} \zeta\right.$ ) acting as a major TG lipase in lipolysis and PNPLA3 $\left(\mathrm{iPLA}_{2} \varepsilon\right.$ ) probably acting as an acyltransferase or transacylase leading to TG accumulation in non-alcoholic fatty liver disease $^{24,48}$. Herein, as part of our ongoing attempts to clarify the biological roles of the $\mathrm{PLA}_{2}$ family using comprehensive gene targeting strategies, we have identified PNPLA1, which represents an ichthyosis-causative gene with unknown function 29 , as an enzyme essential for the biosynthesis of acylceramide, a unique lipid component, the presence of which has long been recognized as prerequisite for normal skin barrier function.

Three abundant lipid groups were almost completely absent in Pnpla $^{-1-}$ epidermis. One of these groups is the acylceramide EOS (and EOP), a key lipid intermediate that is an absolute requirement for formation of the skin barrier and contains saturated, monounsaturated or diunsaturated ULCFA in the $\mathrm{N}$ acyl chain and linoleate in the $\omega$-O-acyl chain. The second group is GlcEOS, a glucosylated form of EOS, which can be stored in lamellar bodies to be secreted into the intercellular space of the SC and then converted back to EOS by the glucosidase GBA. The third group is linoleate-containing OAHFA (OLHFA), as described below. The corresponding accumulation of putative precursors of these three lipid groups, namely $\omega-\mathrm{OH}$ Cer, $\omega$-OH GlcCer and $\omega$ OH ULCFA, in PNPLA1-deficient epidermis provides strong evidence that PNPLAl acts as an $\omega$-O-acyltransferase or transacylase required for acylceramide synthesis. In this regard, the accompaning study by Ohno et al. ${ }^{49}$ has clearly shown that exogenous overexpression of PNPLA1 in cells or PNPLA1reconstituted proteoliposomes promotes acylceramide formation likely as a transacylase and that PNPLA1 mutations associated with ARCI inactivate this transacylase activity.

So far, the order and molecular mechnisms by which ULCFAs and specifically LA are hooked onto the $\omega-\mathrm{OH}$ ULCFAs of (glucosyl)ceramides has not been fully clarified ${ }^{15,21}$. Our new proposed model for epidermal ceramide metabolism is as follows (Fig. 6d): LA is directly tranferred from a linolate-containing TG pool to the $\omega-\mathrm{OH}$ ULCFA moiety by PNPLA1 as a
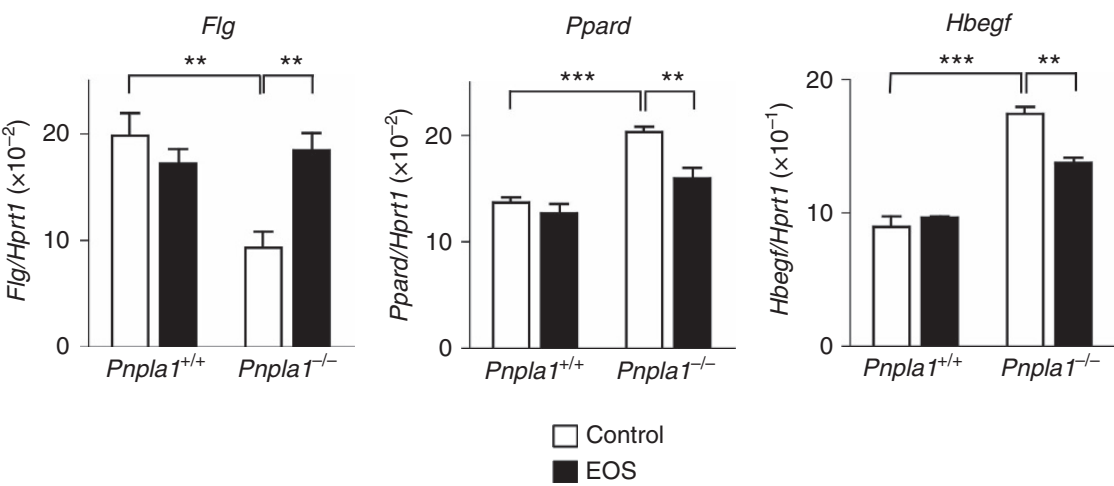

Figure 5 | Cer EOS partially rescues aberrant differentiation of Pnpla1 ${ }^{-1-}$ keratinocytes. qPCR analysis of gene expression in Pnpla1-deficient and control keratinocytes treated with $1.2 \mathrm{mM} \mathrm{CaCl}_{2}$ for $48 \mathrm{~h}$. EOS was added to the culture medium at $10 \mu \mathrm{M}$ for the last $24 \mathrm{~h}$. Data are presented as the mean \pm s.e.m. $\left(n=4 ;{ }^{\star \star} P<0.01\right.$ and $\left.{ }^{\star \star \star} P<0.001\right)$. Results are representative of two experiments. 
a

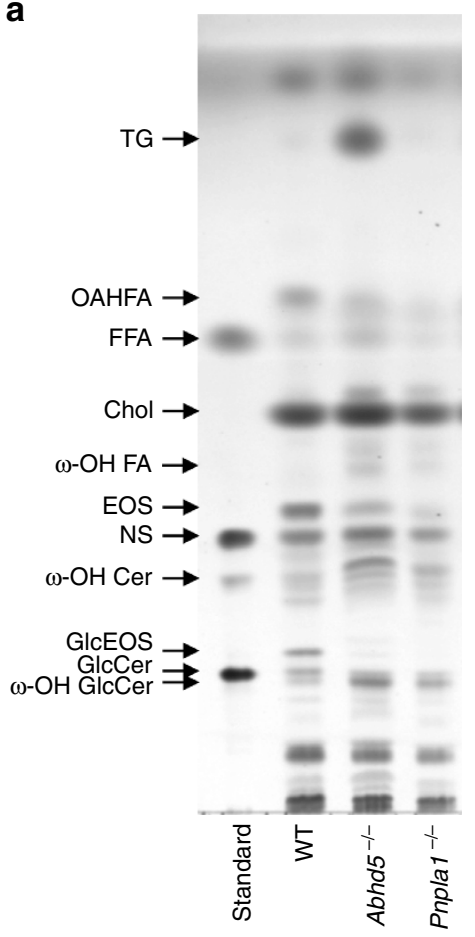

b

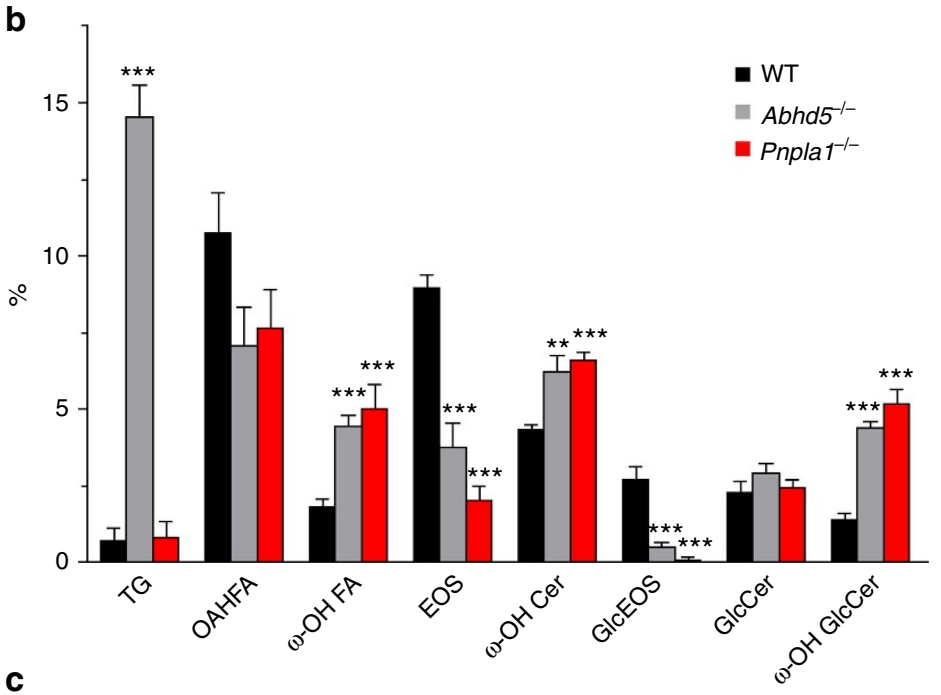

C

\begin{tabular}{|l|c|c|c|c|c|c|c|}
\hline & TG & OAHFA & $\begin{array}{c}\omega \text {-OH } \\
\text { FA }\end{array}$ & EOS & $\begin{array}{c}\omega \text {-OH } \\
\text { Cer }\end{array}$ & $\begin{array}{c}\text { Glc } \\
\text { EOS }\end{array}$ & $\begin{array}{c}\omega \text {-OH } \\
\text { GlcCer }\end{array}$ \\
\hline Abhd5 $5^{-/}$ & $\uparrow \uparrow \uparrow$ & $\downarrow$ & $\uparrow$ & $\downarrow$ & $\uparrow$ & $\downarrow \downarrow \downarrow$ & $\uparrow \uparrow$ \\
\hline Pnpla1 $^{-/}$ & & $\downarrow$ & $\uparrow$ & $\downarrow \downarrow$ & $\uparrow$ & $\downarrow \downarrow \downarrow$ & $\uparrow \uparrow$ \\
\hline
\end{tabular}

d



Figure 6 | Roles of PNPLA1 and ABHD5 in epidermal ceramide metabolism. (a) Representative TLC analysis of lipids extracted from WT, $A b h d 5^{-/-}$, and Pnpla1 ${ }^{-/-}$epidermis at E18.5. (b) Quantification of panel A by densitometric analysis (mean \pm s.e.m., $n=6,5$ and 5 for WT, Abhd5 ${ }^{-/-}$and Pnpla1 ${ }^{-/-}$ mice, respectively; ${ }^{\star \star} P<0.01$ and ${ }^{\star \star \star} P<0.001$ versus WT mice). Cumulative results of three independent experiments are shown. (c) A summary profile for a,b. Up and down arrows represent an increase and a decrease, respectively, in the level of individual lipids in mutant mice relative to WT mice. The number of arrows indicates the relative degree of difference. (d) Schematic diagram showing the proposed role of PNPLA1 in epidermal ceramide metabolism in association with keratinocyte differentiation and skin barrier function. Significantly increased and decreased lipid metabolites in Pnpla ${ }^{-/-}$epidermis are highlighted in red and blue, respectively. Upregulated enzymes are shown in italics. LA derived from TG appears to be esterified at the $\omega$-position of $\omega$-OH ULCFA, $\omega-\mathrm{OH}$ Cer and/or $\omega-\mathrm{OH}$ GlcCer (reactions 1, 2 and 3, respectively) by PNPLA1 transacylase. For details, see text.

CoA-independent transacylase to form OAHFA, EOS and/or GlcEOS. Glucosylation of ceramide occurs in the cis-Golgi apparatus through the action of UDP-glucose ceramide glucosyltransferase, UGCG, and then the resulting GlcEOS is incorporated into lamellar bodies and secreted into the intercellular space of the SC. At the SC interstices, the glucosidase GBA deglycosylates GlcEOS to EOS, which form lipid lamellae together with cholesterol and FFA. Two lipoxygenases, ALOX12B and ALOXE3, sequentallly oxygenate the linoleate moiety in EOS (ref. 50), which then allows a second 
as-yet-unidentified lipase to de-esterify acylceramides. The resulting pool of $\omega-\mathrm{OH}$ Cer can then be covalently linked to the outer suface of the CE, thus foming the CLE.

Although it has been reported that OAHFAs are abundant in the epidermis ${ }^{51}$, the function and origin of this unique class of lipids in the epidermis have remained unknown. It is likely that OAHFAs share a biosynthetic reaction with acylceramides, which also contain an $\mathrm{N}$-acyl chain composed of a particular type of OAHFA, namely linoleate-containing OLHFA. There are at least two possible pathways for OAHFA biosynthesis, either directly via $\omega$-O-esterification of $\omega$-OH ULCFA with LA by PNPLA1 (Route 1 in Fig. 6d) or indirectly via synthesis of acyl(glucosyl)ceramides by PNPLA1 and subsequent hydrolysis by a ceramidase (Route 2 and 3 in Fig. 6d). Whether CERS3 could use OAHFA (or its -CoA form) as a substrate for acylceramide synthesis remains to be determined.

The marked alteration of epidermal structure and function along with impaired acylceramide synthesis in Pnpla1 ${ }^{-1-}$ newborns, accompanied by down-regulation of $\mathrm{CE}$ proteins and up-regulation of EGF ligands, indicate that acylceramide biosynthesis is required for not only the water-impermeable intercellular lipid lamellae in the SC, but also the proper transition from proliferation to terminal differentiation of keratinocytes. The delayed onset of skin phenotypes in Pnpla ${ }^{f / f} K 14-C r e$ mice in comparison with global Pnpla1 ${ }^{-1-}$ mice may be due to incomplete deletion of cutaneous Pnpla1 expression at birth in the former. The increased expression of PPAR $\delta$ in Pnpla $1^{-1}$ - epidermis could explain, at least in part, the induction of a panel of lipid metabolism-related genes associated with ARCI. Indeed, PPAR $\delta$ contributes to up-regulation of ABCA12 and GBA in keratinocytes, and PPAR $\delta$ deficiency decreases lipid metabolism required for lamellar membrane formation and thereby skin barrier function ${ }^{52-54}$. On the other hand, hyperactivation of PPAR $\delta$ enhances keratinocyte proliferation through inducing HB-EGF (ref. 43), an event that is recapitulated in Pnpla $1^{-1-}$ keratinocytes.

It is tempting to speculate that the increased extracellular levels of acylceramide or its derivative(s) at the SG/SC border could provide a critical signal for keratinocyte maturation to corneocytes. In our study using cultured Pnpla $1^{-1-}$ keratinocytes, the supplementation with EOS reversed the decreased expression of filaggrin and increased expression of HB-EGF towards normal levels. In support of this observation, application of synthetic pseudo-acylceramide or GlcEOS recovers diminished barrier function in vivo and promotes maturation of cultured keratinocytes by facilitating cornification and CE formation ${ }^{55,56}$. Moreover, markers for keratinocyte proliferation and differentiation are dysregulated in several other knockout mouse lines deficient in the pathway leading to EOS synthesis, processing or transport (for example, Elovl4 ${ }^{-1-}$, Cers $^{-1-}$, Abhd5 $5^{-1-}$, Abca12 $2^{-1-}$ and epidermal-specific $\left.U g c g^{-1-}\right)^{22,35,41,57,58}$. In contrast, keratinocyte differentiation is not profoundly affected in Alox $12 b^{-1-}$ mice $^{59}$, where protein-bound lipids, but not free ceramides including EOS, are decreased, consistent with the view that the LOX-catalysed oxidation of the linoleate residue in acylceramide is required for subsequent ester hydrolysis and covalent binding of the resultant free $\omega-\mathrm{OH}$ Cer to the $\mathrm{CE}^{50}$. These differences could be explained if differentiated keratinocytes have the ability to sense an extracellular pool of acylceramide or its derivative(s) through a putative receptor, transporter or other way. Nonetheless, the existence of such cross-talk between acylceramide metabolism and transcriptional control of keratinocyte differentiation would be advantageous for the coordinated formation of corneocytes and intercellular lamellar membranes that comprise the SC with competent permeability barrier function, although full understanding of the underlying mechanism needs further elucidation.

Overall, our analyses of epidermal lipids, morphology and permeability barrier function lend strong support to the contention that PNPLA1 is essential for acylceramide synthesis and skin barrier function. Our genetic approach using knockout mice and the biochemical approach by Ohno et al. ${ }^{49}$ complement each other by providing different lines of evidence that prove that PNPLA1 catalyses the $\omega$-O-esterification in acylceramide biosynthesis. While our manuscript was under final review, Grond et al. ${ }^{60}$ also reported that acylceramide biosynthesis was impaired in the skin of another Pnpla1 ${ }^{-1-}$ mouse strain and in human keratinocytes with PNPLA1 mutation, and that topical application of epidermal lipids from WT mice to Pnpla1 ${ }^{-1-}$ skin promoted rebuilding of the CLE. Herein, by means of comprehensive lipidomics, global gene profiling and conditional targeting, we have provided additional insights that the action of PNPLA1 is highly linoleate-selective and keratinocyte-intrinsic. Indeed, $\omega$-O-acyl linoleate in acylceramides and OAHFAs is largely abolished with only partial replacement by other fatty acids in the Pnpla1 ${ }^{-1-}$ epidermis, implying that PNPLA1 selectively utilizes linoleic acid for acylceramide biosynthesis and that the loss of this linoleate specificity causes epidermal barrier defect. Although the catalytic mechanism, subcellular localization and functional regulation of PNPLA1 still remain to be elucidated, the findings obtained from these three complementary studies altogether contribute to a better understanding of the skin barrier formation and ichthyosis development, and should be useful in providing novel therapeutic strategies for treatment of patients with skin barrier disorders.

\section{Methods}

Keratinocyte culture. Mouse primary keratinocytes were isolated as described previously ${ }^{61}$. Briefly, skins of newborn mice were treated with $5 \mathrm{mg} \mathrm{ml}^{-1}$ Dispase (Thermo Fisher) overnight at $4^{\circ} \mathrm{C}$. The epidermis was then mechanically separated from the dermis and incubated with Accutase (Nacalai tesque) for $20 \mathrm{~min}$ at room temperature to collect keratinocytes. Human and mouse progenitors for epidermal keratinocytes were purchased from CELLnTEC and have been tested for mycoplasma by the distributor. These cells were cultured in CnT-Prime medium containing $1 \%(\mathrm{v} / \mathrm{v})$ antibiotic-antimycotic solution (Thermo Fisher). After reaching confluency, the cells were cultured for appropriate periods in CnT-Prime 2D Diff medium supplemented with $1.2 \mathrm{mM} \mathrm{CaCl}_{2}$ to induce keratinocyte differentiation. As required for experiments, Cer EOS ( $N$-(30-Linoleoyloxytriacontanoyl)-sphingosine; Matreya LLC) was dispersed by sonication for $1 \mathrm{~min}$ and then added to the culture. All media were from CELLnTEC.

Mice. Pnpla1-deficient mice, containing a 'knockout-first' allele targeted to the Pnpla1 genomic locus named Pnpla1 ${ }^{\operatorname{tm} 1 a(K O M P) W t s i}$, were generated from a conditional targeting vector obtained from the Knockout Mouse Project resource (KOMP-CSD ID:79620) (ref. 62). Briefly, mouse embryonic stem cells derived from C57BL/6N mice (RENKA) ${ }^{63}$ containing the correctly targeted Pnpla1 locus were injected into blastocysts and transplanted in pseudopregnant mice to generate chimaera mice. Highly (80-90\%) chimeric males were mated with C57BL/6N females, and germ line transmission of the targeted allele was confirmed by PCR. The IRES-LaCZ and Neo cassettes were removed by flippase-mediated excision. The male and female heterozygous mice were intercrossed to obtain homozygous null mice, and littermate WT mice were used as controls. Mice with a floxed allele of Pnpla1 were crossed with transgenic mice for K14 promoter-driven Cre recombinase $^{46}$ to obtain skin-specific Pnpla1 ${ }^{-1}$ mice (Pnpla1 ${ }^{\text {f/f }}$ K14-Cre).

To generate $A b h d 5^{-1-}$ mice, genomic $A b h d 5$ clones were isolated from mouse $129 \mathrm{v} /$ Ev genomic library. A $6.7-\mathrm{kb}$ fragment of an Abhd5 clone was subcloned into a targeting vector with exon 1 being replaced by the PGK-Neo cassette. The targeting vector was introduced into $129 \mathrm{~Sv} / \mathrm{Ev}$ embryonic stem cells and a correctly targeted embryonic stem cell line was injected into blastocysts, resulting in the gene-targeted mouse strain. Heterozygotes were backcrossed onto C57BL/6 J background for at least five generations and then intercrossed to obtain homozygous null mice.

Genotyping of offspring was performed by PCR of tail-snip DNA using genotyping primers (Supplementary Table 1). Animals were fed ad libitum (CE2, Clea Japan), had free access to water, and were kept on a 12:12-h light:dark cycle in single cages. All experimental procedures involving animals in this study were approved by the Institutional Animal Care and Use Committees of Tokyo Metropolitan Institute of Medical Science and Nagoya University and were 
conducted in accordance with the Japanese Guide for the Care and Use of Laboratory Animals.

Skin permeability assays. Toluidine blue staining of newborn mice was described previously ${ }^{22}$. In brief, newborns were anesthetized, dehydrated in methanol, washed in PBS and stained for $30 \mathrm{~min}$ in $0.1 \%(\mathrm{w} / \mathrm{v})$ toluidine blue/PBS. After washing in PBS, the pups were photographed. TEWL was measured using a Tewameter TM300 with a 2-mm-diameter adaptor (Courage-Khazaka Electronics, Germany). Measurements were performed after calibration of the device at room temperature with minimized influence of air turbulence, and the results were recorded when the TEWL values were stabilized $30-45 \mathrm{~s}$ after probe placement.

Histology and immunohistochemistry. Skins were frozen and embedded in OCT compound, or fixed overnight with buffered $4 \%(\mathrm{w} / \mathrm{v})$ paraformaldehyde at $4{ }^{\circ} \mathrm{C}$ and embedded in paraffin. Skin sections $(5 \mu \mathrm{m})$ were stained with hematoxylin and eosin or processed for immunoreactions. For immunofluorescent staining, the deparaffinized sections were blocked with PBS containing $10 \%$ (w/v) Block Ace (Sumitomo Dainippon Pharma), incubated with primary antibodies overnight at $4^{\circ} \mathrm{C}$ and with secondary Alexa Fluor antibodies at room temperature for $1 \mathrm{~h}$. Rabbit polyclonal antibody against PNPLA1 was raised against a synthetic peptide GPPVEDLGPERPTATGSP as an immunogen and used at 1:2,000 dilution. Other primary antibodies used at 1:1,000 dilution were rabbit antibodies against filaggrin, keratin 1, keratin 5, keratin 6 and loricrin (PRB-417P, PRB-149P, PRB-160P, PRB-169P and PRB-145P; Covance). Secondary antibodies used at 1:1,000 dilution were Alexa Fluor 546 goat anti-rabbit IgG and Alexa Fluor 633 goat anti-rabbit IgG (Thermo Fisher). For double immunostaining, rabbit polyclonal antibodies were pre-labelled with Alexa Fluor 488 or Alexa Fluor 555 using the Zenon Rabbit IgG Labeling Kits (Thermo Fisher). After immunoreactions and counterstaining with DAPI (Vector Laboratories), the slides were visualized using an LSM710 confocal microscope (Zeiss). For immunostaining of adult mouse skin, the sections were incubated with anti-PNPLA1 antibody, treated with biotinylated anti-rabbit antibody (BA-1000, Vector Laboratories), and then processed with an avidin-biotinperoxidase system (Vectastain Elite $\mathrm{ABC}$ kit, Vector Laboratories) and diaminobenzidine, followed by counterstaining with hematoxylin. Lipids were visualized on cryosections by staining with Nile Red $\left(5 \mathrm{mg} \mathrm{ml}^{-1}\right.$ in $75 \%$ (v/v) glycerol, Wako).

Electron microscopy. Neonatal mouse skin samples were fixed in $5 \%(\mathrm{w} / \mathrm{v})$ glutaraldehyde solution, post-fixed in $0.5 \%(\mathrm{w} / \mathrm{v})$ ruthenium tetroxide $\left(\mathrm{RuO}_{4}\right)$, dehydrated and embedded in Epon812 (TAAB Laboratories). All the samples were ultra-thin sectioned at a thickness of $70 \mathrm{~nm}$, and stained with uranyl acetate and lead citrate. Photographs were taken using a JEM1400 transmission electron microscope (JEOL Ltd.).

Quantitative PCR. Total RNA was extracted with TRIzol (Thermo Fisher) and was reverse-transcribed into cDNA using ReverTra Ace qPCR RT Master Mix (Toyobo) in accordance with the manufacturer's instructions. qPCR reactions were performed on a LightCycler480-II (Roche) using THUNDERBIRD Probe qPCR Mix (Toyobo). The sequences of primers designed to be compatible with the Roche Universal Probe Library (UPL) are provided in Supplementary Tables 2 and 3. The hydrolysis probe used in the assay was labelled with a fluorescein-based reporter dye (FAM) and a non-fluorescent quencher. Cycling conditions were the following: $95^{\circ} \mathrm{C}$ for $15 \mathrm{~min}$ (one cycle), $95^{\circ} \mathrm{C}$ for $15 \mathrm{~s}$ and $60^{\circ} \mathrm{C}$ for $1 \mathrm{~min}$ ( $40 \mathrm{cycles}$ ). A total of 1-2 $\mu \mathrm{l}$ of cDNA per sample was used for the quantification of endogenous mRNA levels. Expression levels were normalized to Hprt1 or RPL13A.

Microarray. Total RNA extracted from P0 newborns was purified using a RNeasy Mini Kit (QIAGEN). The quality of RNA was assessed with a 2100 Bioanalyzer (Agilent Technologies). Fluorescently labelled antisense RNA (cRNA targets) were synthesized with a Low Input QuickAmp Labeling Kit according to the manufacturer's protocol (Agilent Technologies). Samples were hybridized to the Mouse Gene Expression 4x44K v2 Microarray (G4846A, Agilent Technologies), washed, and then scanned using a SureScan Microarray Scanner (Agilent Technologies). Microarray data were analysed with Feature Extraction software (Agilent Technologies) and then imported into GeneSpring GX software (Agilent Technologies). Signal intensities were normalized by global normalization.

Lipid analysis. After subcutaneous tissue was removed by scraping on ice, skin pieces were incubated in phosphate-buffered saline at $60^{\circ} \mathrm{C}$ for $1 \mathrm{~min}$ or in phosphate-buffered saline containing $1.5 \mathrm{mg} \mathrm{ml}^{-1}$ dispase (Invitrogen) at $4^{\circ} \mathrm{C}$ overnight, and the epidermis was peeled from the dermis. The isolated epidermis was vortex-homogenized with steel beads in $1 \mathrm{ml}$ of methanol using beads crusher $\mu \mathrm{T}-01$ (TITEC). Free lipids were extracted by a modified Folch method ${ }^{64,65}$ or with a series of mixtures of chloroform/methanol 2:1, 1:1 and 1:2 (v/v). Combined organic phases were washed once with $0.5 \mathrm{ml}$ of $0.88 \% \mathrm{KCl}$ and twice with distilled water and then dried with a nitrogen stream. Covalently bound lipids were extracted by incubating the remaining tissue in $2 \mathrm{ml}$ of $1 \mathrm{M} \mathrm{NaOH}$ in $90 \%(\mathrm{v} / \mathrm{v})$ methanol at $60^{\circ} \mathrm{C}$ for $2 \mathrm{~h}$. After adjusting the $\mathrm{pH}$ to 6 with $3 \mathrm{M} \mathrm{HCl}$, lipids were extracted twice with chloroform. The organic layer was washed twice with distilled water. The amount of dry lipids was calculated by subtracting the weight of the empty vials.

TLC. Epidermal lipids corresponding to $5 \mathrm{mg}$ dry weight were separated by TLC (Silica gel 60, Merck) with the following solvent sequence: 1) chloroform/methanol/water $(40: 10: 1)$ to $2 \mathrm{~cm} ; 2)$ chloroform/methanol/water $(40: 10: 1)$ to $5 \mathrm{~cm}: 3)$ chloroform/methanol/acetic acid $(47: 2: 0.5)$ to $8.5 \mathrm{~cm} ; 4)$ n-hexane/diethyl ether/ acetic acid (65:35:1) to the top of the plate. Lipids were visualized after treatment with $5 \%(\mathrm{w} / \mathrm{v}) \mathrm{CuSO}_{4}$ in $15 \%(\mathrm{v} / \mathrm{v}) \mathrm{H}_{3} \mathrm{PO}_{4}$ and heating to $180^{\circ} \mathrm{C}$ for $10 \mathrm{~min}$. Lipid classifications were performed by comparison with authentic lipid standards or by LC-MS/MS analysis. The intensities of bands were quantified by densitometry using LAS-4000 imaging system (Fuji Film) and JustTLC software (Version 4.0.3, Sweday).

MS analysis. LC-MS/MS and LC-MS were used for identification and quantification of epidermal lipids. Non-targeted lipidomics analysis ${ }^{65,66}$ were performed with minor modifications. Briefly, dried total lipid extracts were re-dissolved in $50 \mu \mathrm{l}$ of chloroform:methanol $(2: 1, \mathrm{v} / \mathrm{v})$ and $2 \mu \mathrm{l}$ of samples were separated by an ACQUITY UPLC BEH C18 column $(50 \times 2.1 \mathrm{~mm}$ i.d., particle size $1.7 \mu \mathrm{m}$, Waters $)$ at a flow rate of $300 \mu \mathrm{min}^{-1}$ at $45^{\circ} \mathrm{C}$ using an ACQUITY UPLC system (Waters) equipped with a binary pump and automatic sample injector. Solvent A consisted of acetonitrile/methanol/water (20:20:60, v/v/v) and solvent B was isopropanol, both containing $5 \mathrm{mM}$ ammonium acetate. The solvent composition started at $100 \% \mathrm{~A}$ for the first $1 \mathrm{~min}$ and was changed linearly to $64 \% \mathrm{~B}$ at $7.5 \mathrm{~min}$, where it was held for $4.5 \mathrm{~min}$. The gradient was increased linearly to $82.5 \% \mathrm{~B}$ at $12.5 \mathrm{~min}$, followed by $85 \% \mathrm{~B}$ at $19 \mathrm{~min}$ and $95 \% \mathrm{~B}$ at $20 \mathrm{~min}$ before re-equilibrating the column with $100 \%$ A for $5 \mathrm{~min}$. Qualitative and quantitative analysis of lipids was performed by MS and data-dependent MS/MS acquisition with a scan range of $\mathrm{m} / \mathrm{z}$ 70-1250 using a Triple TOF $5600^{+}$System (AB SCIEX) in the negative and positive ion mode. Raw data files from the TOF-MS were converted to MGF files using the program $\mathrm{AB}$ SCIEX MS converter for subsequent quantitative analysis with 2DICAL (Mitsui Knowledge Industry). Identification of molecular species was accomplished by comparison with retention times and MS/MS spectra with commercially available standards or reference samples.

Ceramides, VLCFAs, and cholesterol were quantified by LC-MS using an Agilent 1100 Series LC/MSD SL system equipped with a multi-ion source, ChemStation software, an autosampler and an L-column ODS $(150 \times 2.1 \mathrm{~mm}$ i.d.; Chemicals Evaluation and Research Institute $)^{20,67}$. Briefly, the lipid extracts supplemented with the internal standard C17:0 ceramide were dried under a nitrogen stream and then were dissolved in chloroform/methanol/2-propanol $(10: 45: 45, \mathrm{v} / \mathrm{v} / \mathrm{v})$. Lipid sample of $20 \mu \mathrm{l}$ was injected and separated by reversedphase chromatography at a flow rate of $0.2 \mathrm{ml} \mathrm{min}^{-1}$ using a binary gradient solvent system: Solvent $C$ consisted of methanol/water $(1: 1, v / v)$ and solvent $D$ was 2-propanol, both containing $5 \mathrm{mM}$ acetic acid and $10 \mathrm{mM}$ ammonium acetate. The column temperature was maintained at $40^{\circ} \mathrm{C}$ and the mobile phases were consecutively programmed as follows: $0-1 \mathrm{~min}, 20 \% \mathrm{D} ; 1-2 \mathrm{~min}$, gradient to $60 \%$ $\mathrm{D} ; 2-30 \mathrm{~min}$, gradient to $100 \% \mathrm{D} ; 30-35 \mathrm{~min}, 100 \% \mathrm{D} ; 35-45 \mathrm{~min}, 20 \% \mathrm{D}$. MS parameters were as follows: negative ion mode, flow of heated dry nitrogen gas $4.01 \mathrm{~min}^{-1}$, nebulizer gas pressure $60 \mathrm{psi}$, heater temperature of nitrogen gas $350{ }^{\circ} \mathrm{C}$, vaporizer temperature $200^{\circ} \mathrm{C}$, capillary voltage $4,000 \mathrm{~V}$, charging voltage $2,000 \mathrm{~V}$ and fragmenter voltage $200 \mathrm{~V}$. Each ceramide species was detected by selected ion monitoring as $\mathrm{m} / z\left[\mathrm{M}+\mathrm{CH}_{3} \mathrm{COO}\right]^{-}$.

Analysis of phospholipids and MCFAs was performed using a 4000 QTRAP quadrupole-linear ion trap hybrid MS (AB Sciex) with liquid chromatography (LC-20AP; Shimadzu) ${ }^{33}$. The internal standard mixture added to each sample (equivalent to $2 \mathrm{mg}$ dry weight skin) contained $400 \mathrm{pmol}$ of PE 28:0,100 pmol of phosphatidylcholine (PC) 28:0 and $100 \mathrm{pmol}$ of lysoPC 17:0. Sample $(10 \mu \mathrm{l})$ was injected by an autosampler and separated using a Develosil C30-UG column $(150 \times 1.0 \mathrm{~mm}$ i.d., particle size $3 \mu \mathrm{m}$, Nomura Chemical) by a step gradient at a flow rate of $80 \mu \mathrm{min}^{-1}$ at $50^{\circ} \mathrm{C}$. Solvent E consisted of acetonitrile/methanol/ water $(1: 1: 1, \mathrm{v} / \mathrm{v} / \mathrm{v})$ and solvent $\mathrm{F}$ was 2 -propanol, both containing $5 \mu \mathrm{M}$ phosphoric acid and $1 \mathrm{mM}$ ammonium formate. Lipid peaks were identified according to retention times and multiple reaction monitoring transitions, and quantified by comparison with standard curves using the peak area ratio method.

Covalently bound ceramide extracts were separated by straight-phase HPLC and analysed using Thermo Finnigan DSQ instrument with an APCI ion source operated in positive mode. Sample of $5 \mu \mathrm{l}$ was injected into a TLC Advantage Silica column $(250 \times 4.6 \mathrm{~mm}$ i.d., $5 \mu \mathrm{m}$ particle size, $150 \AA$ pore size; Thomson Instrument Company) and separated with hexane/isopropanol/acetic acid $(90: 10: 0.1, \mathrm{vol} / \mathrm{vol} / \mathrm{vol})$ at a flow rate of $1 \mathrm{ml} \mathrm{min}^{-1}$. Settings in the DSQ were as follows: capillary temperature $275^{\circ} \mathrm{C}$, ion transfer voltage $2,000 \mathrm{~V}$, vaporizer temperature $450^{\circ} \mathrm{C}$, gas 1 set at 50 , gas 2 set at 5 , electron energy $70 \mathrm{eV}$ and full scan $m / z$ range $400-1,400$. The spectra were obtained in full scan mode.

Statistical analysis. Sample sizes were chosen based on previous experience in our laboratory. The experiments were performed and analysed in non-randomized and non-blinded fashion. No data were excluded from the analysis. Significance was determined by unpaired two-tailed Student's $t$-test. Variance was similar 
between the groups that were statistically compared. A $P$ value of $<0.05$ was considered statistically significant. All the data were presented as mean \pm s.e.m.

Data availability. The data that support the findings of this study are available from the corresponding author on request. The microarray data can be accessed at the GEO repository under the accession number GSE87682.

\section{References}

1. Breiden, B. \& Sandhoff, K. The role of sphingolipid metabolism in cutaneous permeability barrier formation. Biochim. Biophys. Acta 1841, 441-452 (2014).

2. Feingold, K. R. Thematic review series: skin lipids. The role of epidermal lipids in cutaneous permeability barrier homeostasis. J. Lipid Res. 48, 2531-2546 (2007).

3. Elias, P. M., Williams, M. L., Holleran, W. M., Jiang, Y. J. \& Schmuth, M. Pathogenesis of permeability barrier abnormalities in the ichthyoses: inherited disorders of lipid metabolism. J. Lipid Res. 49, 697-714 (2008).

4. Segre, J. A. Epidermal barrier formation and recovery in skin disorders. J. Clin. Invest. 116, 1150-1158 (2006).

5. Guttman-Yassky, E., Nograles, K. E. \& Krueger, J. G. Contrasting pathogenesis of atopic dermatitis and psoriasis-part I: clinical and pathologic concepts. J. Allergy Clin. Immunol. 127, 1110-1118 (2011).

6. De Benedetto, A., Kubo, A. \& Beck, L. A. Skin barrier disruption: a requirement for allergen sensitization? J. Invest. Dermatol. 132, 949-963 (2012).

7. van Smeden, J. \& Bouwstra, J. A. Stratum corneum lipids: their role for the skin barrier function in healthy subjects and atopic dermatitis patients. Curr. Probl. Dermatol. 49, 8-26 (2016).

8. Elias, P. M. et al. Formation and functions of the corneocyte lipid envelope (CLE). Biochim. Biophys. Acta 1841, 314-318 (2014).

9. Tsuruta, D., Green, K. J., Getsios, S. \& Jones, J. C. The barrier function of skin: how to keep a tight lid on water loss. Trends Cell Biol. 12, 355-357 (2002).

10. Candi, E., Schmidt, R. \& Melino, G. The cornified envelope: a model of cell death in the skin. Nat. Rev. Mol. Cell. Biol. 6, 328-340 (2005).

11. Rabionet, M., Gorgas, K. \& Sandhoff, R. Ceramide synthesis in the epidermis. Biochim. Biophys. Acta 1841, 422-434 (2014).

12. van Smeden, J., Janssens, M., Gooris, G. S. \& Bouwstra, J. A. The important role of stratum corneum lipids for the cutaneous barrier function. Biochim. Biophys. Acta 1841, 295-313 (2014).

13. Masukawa, Y. et al. Comprehensive quantification of ceramide species in human stratum corneum. J. Lipid. Res. 50, 1708-1719 (2009).

14. t'Kindt, R. et al. Profiling and characterizing skin ceramides using reversedphase liquid chromatography-quadrupole time-of-flight mass spectrometry. Anal. Chem. 84, 403-411 (2012).

15. Uchida, Y. \& Holleran, W. M. Omega- $O$-acylceramide, a lipid essential for mammalian survival. J. Dermatol. Sci. 51, 77-87 (2008).

16. Wertz, P. W. Lipids and barrier function of the skin. Acta Derm. Venereol. Suppl. (Stockh.) 208, 7-11 (2000).

17. Wertz, P. W., Cho, E. S. \& Downing, D. T. Effect of essential fatty acid deficiency on the epidermal sphingolipids of the rat. Biochim. Biophys. Acta 753, 350-355 (1983).

18. Eckl, K. M. et al. Impaired epidermal ceramide synthesis causes autosomal recessive congenital ichthyosis and reveals the importance of ceramide acyl chain length. J. Invest. Dermatol. 133, 2202-2211 (2013).

19. Fischer, J. Autosomal recessive congenital ichthyosis. J. Invest. Dermatol. 129, 1319-1321 (2009).

20. Ohno, Y. et al. Essential role of the cytochrome P450 CYP4F22 in the production of acylceramide, the key lipid for skin permeability barrier formation. Proc. Natl Acad. Sci. USA 112, 7707-7712 (2015).

21. Radner, F. P. \& Fischer, J. The important role of epidermal triacylglycerol metabolism for maintenance of the skin permeability barrier function. Biochim. Biophys. Acta 1841, 409-441 (2014).

22. Radner, F. P. et al. Growth retardation, impaired triacylglycerol catabolism, hepatic steatosis, and lethal skin barrier defect in mice lacking comparative gene identification-58 (CGI-58). J. Biol. Chem. 285, 7300-7311 (2010).

23. Uchida, Y. et al. Neutral lipid storage leads to acylceramide deficiency, likely contributing to the pathogenesis of Dorfman-Chanarin syndrome. J. Invest. Dermatol. 130, 2497-2499 (2010).

24. Zimmermann, R. et al. Fat mobilization in adipose tissue is promoted by adipose triglyceride lipase. Science 306, 1383-1386 (2004).

25. Schweiger, M., Lass, A., Zimmermann, R., Eichmann, T. O. \& Zechner, R. Neutral lipid storage disease: genetic disorders caused by mutations in adipose triglyceride lipase/PNPLA2 or CGI-58/ABHD5. Am. J. Physiol. Endocrinol. Metab. 297, E289-E296 (2009).

26. Kienesberger, P. C., Oberer, M., Lass, A. \& Zechner, R. Mammalian patatin domain containing proteins: a family with diverse lipolytic activities involved in multiple biological functions. J. Lipid. Res. 50, S63-S68 (2009).
27. Ahmad, F. et al. A novel missense variant in the PNPLAl gene underlies congenital ichthyosis in three consanguineous families. J. Eur. Acad. Dermatol. Venereol. 30, e210-e213 (2016).

28. Fachal, L. et al. Identification of a novel PNPLA1 mutation in a Spanish family with autosomal recessive congenital ichthyosis. Br. J. Dermatol. 170, 980-982 (2014).

29. Grall, A. et al. PNPLA1 mutations cause autosomal recessive congenital ichthyosis in golden retriever dogs and humans. Nat. Genet. 44, 140-147 (2012).

30. Miki, Y. et al. Lymphoid tissue phospholipase $\mathrm{A}_{2}$ group IID resolves contact hypersensitivity by driving antiinflammatory lipid mediators. J. Exp. Med. 210, 1217-1234 (2013).

31. Sato, H. et al. The adipocyte-inducible secreted phospholipases PLA2G5 and PLA2G2E play distinct roles in obesity. Cell Metab. 20, 119-132 (2014).

32. Taketomi, Y. et al. Mast cell maturation is driven via a group III phospholipase $\mathrm{A}_{2}$-prostaglandin $\mathrm{D}_{2}$-DP1 receptor paracrine axis. Nat. Immunol. 14, 554-563 (2013).

33. Yamamoto, K. et al. The role of group IIF-secreted phospholipase $A_{2}$ in epidermal homeostasis and hyperplasia. J. Exp. Med. 212, 1901-1919 (2015).

34. Djalilian, A. R. et al. Connexin 26 regulates epidermal barrier and wound remodeling and promotes psoriasiform response. J. Clin. Invest. 116, 1243-1253 (2006).

35. Amen, N. et al. Differentiation of epidermal keratinocytes is dependent on glucosylceramide:ceramide processing. Hum. Mol. Genet. 22, 4164-4179 (2013).

36. Hwang, J. et al. Epidermal ablation of Dlx3 is linked to IL-17-associated skin inflammation. Proc. Natl Acad. Sci. USA 108, 11566-11571 (2011).

37. Lessard, J. C. et al. Keratin 16 regulates innate immunity in response to epidermal barrier breach. Proc. Natl Acad. Sci. USA 110, 19537-19542 (2013).

38. Nagarajan, P. et al. Ets1 blocks terminal differentiation of keratinocytes and induces expression of matrix metalloproteases and innate immune mediators. J. Cell Sci. 123, 3566-3575 (2010).

39. Bergboer, J. G. et al. Psoriasis risk genes of the late cornified envelope-3 group are distinctly expressed compared with genes of other LCE groups. Am. J. Pathol. 178, 1470-1477 (2011).

40. Komatsu, N. et al. Human tissue kallikrein expression in the stratum corneum and serum of atopic dermatitis patients. Exp. Dermatol. 16, 513-519 (2007).

41. Akiyama, M. The roles of ABCA12 in epidermal lipid barrier formation and keratinocyte differentiation. Biochim. Biophys. Acta 1841, 435-440 (2014).

42. Tran, W. T. et al. EGFR regulation of epidermal barrier function. Physiol. Genom. 44, 455-469 (2012).

43. Romanowska, M. et al. PPAR $\delta$ enhances keratinocyte proliferation in psoriasis and induces heparin-binding EGF-like growth factor. J. Invest. Dermatol. 128, 110-124 (2008).

44. Motta, S. et al. Ceramide composition of the psoriatic scale. Biochim. Biophys. Acta 1182, 147-151 (1993).

45. Robson, K. J., Stewart, M. E., Michelsen, S., Lazo, N. D. \& Downing, D. T. 6-Hydroxy-4-sphingenine in human epidermal ceramides. J. Lipid. Res. 35, 2060-2068 (1994).

46. Li, M. et al. Skin abnormalities generated by temporally controlled RXR $\alpha$ mutations in mouse epidermis. Nature 407, 633-636 (2000).

47. Murakami, M. et al. Recent progress in phospholipase $\mathrm{A}_{2}$ research: from cells to animals to humans. Prog. Lipid. Res. 50, 152-192 (2011).

48. Kumari, M. et al. Adiponutrin functions as a nutritionally regulated lysophosphatidic acid acyltransferase. Cell Metab. 15, 691-702 (2012).

49. Ohno, Y., Kamiyama, N., Nakamichi, S. \& Kihara, A. PNPLA1 is a transacylase essential for the generation of the skin barrier lipid $\omega$-O-acylceramide. Nat. Commun. 8, 14610 (2017).

50. Muñoz-Garcia, A., Thomas, C. P., Keeney, D. S., Zheng, Y. \& Brash, A. R. The importance of the lipoxygenase-hepoxilin pathway in the mammalian epidermal barrier. Biochim. Biophys. Acta 1841, 401-408 (2014).

51. McMahon, A., Butovich, I. A. \& Kedzierski, W. Epidermal expression of an Elovl4 transgene rescues neonatal lethality of homozygous Stargardt disease-3 mice. J. Lipid. Res. 52, 1128-1138 (2011).

52. Feingold, K. R. \& Jiang, Y. J. The mechanisms by which lipids coordinately regulate the formation of the protein and lipid domains of the stratum corneum: Role of fatty acids, oxysterols, cholesterol sulfate and ceramides as signaling molecules. Dermatoendocrinology 3, 113-118 (2011).

53. Jiang, Y. J. et al. Ceramide stimulates ABCA12 expression via peroxisome proliferator-activated receptor $\delta$ in human keratinocytes. J. Biol. Chem. 284, 18942-18952 (2009).

54. Man, M. Q. et al. Basis for improved permeability barrier homeostasis induced by PPAR and LXR activators: liposensors stimulate lipid synthesis, lamellar body secretion, and post-secretory lipid processing. J. Invest. Dermatol. 126, 386-392 (2006).

55. Uchida, Y., Hamanaka, S., Matsuda, K., Mimura, K. \& Otsuka, F. Effect of a chemically-synthesized acylglucosylceramide, epidermoside, on normal human keratinocyte differentiation. J. Dermatol. Sci. 12, 64-68 (1996). 
56. Imokawa, G. et al. Pseudo-acylceramide with linoleic acid produces selective recovery of diminished cutaneous barrier function in essential fatty aciddeficient rats and has an inhibitory effect on epidermal hyperplasia. J. Clin. Invest. 94, 89-96 (1994).

57. Li, W. et al. Depletion of ceramides with very long chain fatty acids causes defective skin permeability barrier function, and neonatal lethality in ELOVL4 deficient mice. Int. J. Biol. Sci. 3, 120-128 (2007).

58. Jennemann, R. et al. Loss of ceramide synthase 3 causes lethal skin barrier disruption. Hum. Mol. Genet. 21, 586-608 (2012).

59. Epp, N. et al. 12R-lipoxygenase deficiency disrupts epidermal barrier function. I. Cell Biol. 177, 173-182 (2007)

60. Grond, S. et al. PNPLA1 deficiency in mice and humans leads to a defect in the synthesis of omega-O-acylceramides. J. Invest. Dermatol. 137, 394-402 (2017).

61. Caldelari, R. \& Müller, J. Short- and long-term cultivation of embryonic and neonatal murine keratinocytes. Methods Mol. Biol. 633, 125-138 (2010).

62. Skarnes, W. C. et al. A conditional knockout resource for the genome-wide study of mouse gene function. Nature 474, 337-342 (2011).

63. Mishina, M. \& Sakimura, K. Conditional gene targeting on the pure C57BL/6 genetic background. Neurosci. Res. 58, 105-112 (2007).

64. Folch, J., Lees, M. \& Sloane Stanley, G. H. A simple method for the isolation and purification of total lipides from animal tissues. J. Biol. Chem. 226, 497-509 (1957).

65. Ikeda, K. in Bioactive Lipid Mediators: current Reviews and Protocols, (eds Yokomizo, T. \& Murakami, M.), 349-356 (Springer, 2015).

66. Takatani, N. et al. Essential role of acyl-ACP synthetase in acclimation of the cyanobacterium Synechococcus elongatus strain PCC 7942 to high-light conditions. Plant Cell Physiol. 56, 1608-1615 (2015).

67. Masukawa, Y. et al. Characterization of overall ceramide species in human stratum corneum. J. Lipid. Res. 49, 1466-1476 (2008).

\section{Acknowledgements}

We thank Drs A. Naoe, H. Tsujimura and J. Ishikawa (Kao Corporation, Japan) for lipid analysis, Drs I. Kawashima (Tokyo Metropolitan Institute of Medical Science, Japan) and A. Yamashita (Teikyo University, Japan) for technical advice, Drs S. Arata and A. Ohazama (Showa University, Japan) for providing K14-Cre transgenic mice, and the other members of the laboratory for discussions and comments. This work was supported by JSPS KAKENHI Grant Numbers JP15H05905, JP15K14957, JP16H02613 (to M.M.) and JP15K15094 (to T.H.), AMED-CREST from Japan Agency for Medical Research and Development (to T.H. and M.M.), Kao foundation (to M.M.) and NIH grant AR51968 (to A.R.B.).

\section{Author contributions}

T.H. and M.M. designed experiments. T.H., T.A., A.K., Y.S., A.S., H.T., K.Y., Y.N. and A.M.-G., performed the experiments. T.H., T.A., Y.S., K.M., K.F., A.R.B., K.I. Makoto Arita and Masashi Akiyama analysed the data. T.O. and C.T. designed and constructed mutant mice. T.H. and M.M. wrote the manuscript with input from all the other authors.

\section{Additional information}

Supplementary Information accompanies this paper at http://www.nature.com/ naturecommunications

Competing financial interests: The authors declare no competing financial interests.

Reprints and permission information is available online at http://npg.nature.com/ reprintsandpermissions/

How to cite this article: Hirabayashi, T. et al. PNPLA1 has a crucial role in skin barrier function by directing acylceramide biosynthesis. Nat. Commun. 8, 14609 doi: $10.1038 /$ ncomms14609 (2017).

Publisher's note: Springer Nature remains neutral with regard to jurisdictional claims in published maps and institutional affiliations.

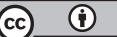

This work is licensed under a Creative Commons Attribution 4.0 International License. The images or other third party material in this article are included in the article's Creative Commons license, unless indicated otherwise in the credit line; if the material is not included under the Creative Commons license, users will need to obtain permission from the license holder to reproduce the material. To view a copy of this license, visit http://creativecommons.org/licenses/by/4.0/

(C) The Author(s) 2017 